\title{
X-Ray and Radio Observations of the Magnetar SGR J1935+2154 during Its 2014, 2015, and 2016 Outbursts
}

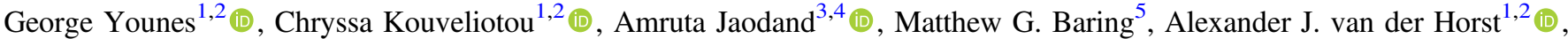 \\ Alice K. Harding ${ }^{6}$ (1) Jason W. T. Hessels ${ }^{3,4}$ (1) , Neil Gehrels ${ }^{6,12}$, Ramandeep Gill ${ }^{7}$ (1) , Daniela Huppenkothen ${ }^{8,9}$ (i), \\ Jonathan Granot ${ }^{7}$ (1) Ersin Göğüs ${ }^{10}$ (10), and Lin $\operatorname{Lin}^{11}$ \\ ${ }^{1}$ Department of Physics, The George Washington University, Washington, DC 20052, USA; gyounes@gwu.edu \\ ${ }^{2}$ Astronomy, Physics and Statistics Institute of Sciences (APSIS), The George Washington University, Washington, DC 20052, USA \\ ${ }^{3}$ ASTRON, the Netherlands Institute for Radio Astronomy, Postbus 2, 7990 AA Dwingeloo, The Netherlands \\ ${ }^{4}$ Astronomical Institute Anton Pannekoek, University of Amsterdam, 1098XH, Amsterdam, The Netherlands \\ ${ }^{5}$ Department of Physics and Astronomy, Rice University, MS-108, P.O. Box 1892, Houston, TX 77251, USA \\ ${ }^{6}$ Astrophysics Science Division, NASA Goddard Space Flight Center, Greenbelt, MD 20771, USA \\ ${ }^{7}$ Department of Natural Sciences, The Open University of Israel, 1 University Road, P.O. Box 808, Raánana 43537, Israel \\ ${ }^{8}$ Center for Data Science, New York University, 726 Broadway, 7th Floor, New York, NY 10003, USA \\ ${ }^{9}$ Center for Cosmology and Particle Physics, Department of Physics, New York University, 4 Washington Place, New York, NY 10003, USA \\ ${ }^{10}$ Sabanc1 University, Orhanl1-Tuzla, İstanbul 34956, Turkey \\ ${ }^{11}$ Department of Astronomy, Beijing Normal University, Beijing 100875, China \\ Received 2017 February 14; revised 2017 August 28; accepted 2017 August 29; published 2017 September 25
}

\begin{abstract}
We analyzed broadband X-ray and radio data of the magnetar SGR J1935+2154 taken in the aftermath of its 2014,2015 , and 2016 outbursts. The source soft X-ray spectrum $<10 \mathrm{keV}$ is well described with a blackbody +power-law $(\mathrm{BB}+\mathrm{PL})$ or 2BB model during all three outbursts. Nuclear Spectroscopic Telescope Array observations revealed a hard X-ray tail, with a PL photon index $\Gamma=0.9$, extending up to $50 \mathrm{keV}$, with flux comparable to the one detected $<10 \mathrm{keV}$. Imaging analysis of Chandra data did not reveal small-scale extended emission around the source. Following the outbursts, the total $0.5-10 \mathrm{keV}$ flux from SGR J1935+2154 increased in concordance to its bursting activity, with the flux at activation onset increasing by a factor of $\sim 7$ following its strongest 2016 June outburst. A Swift/X-Ray Telescope observation taken 1.5 days prior to the onset of this outburst showed a flux level consistent with quiescence. We show that the flux increase is due to the $\mathrm{PL}$ or hot $\mathrm{BB}$ component, which increased by a factor of 25 compared to quiescence, while the cold $\mathrm{BB}$ component $k T=0.47 \mathrm{keV}$ remained more or less constant. The 2014 and 2015 outbursts decayed quasiexponentially with timescales of $\sim 40$ days, while the stronger 2016 May and June outbursts showed a quick short-term decay with timescales of about four days. Our Arecibo radio observations set the deepest limits on the radio emission from a magnetar, with a maximum flux density limit of $14 \mu \mathrm{Jy}$ for the $4.6 \mathrm{GHz}$ observations and $7 \mu \mathrm{Jy}$ for the $1.4 \mathrm{GHz}$ observations. We discuss these results in the framework of the current magnetar theoretical models.
\end{abstract}

Key words: radio continuum: stars - stars: individual (SGR J1935+2154) - stars: magnetars - stars: neutron X-rays: stars

\section{Introduction}

A subset of isolated neutron stars (NSs), dubbed magnetars, show peculiar rotational properties with low spin periods $P$ in the range of $2-12 \mathrm{~s}$ and large spin-down rates $\dot{P}$ of the order of $10^{-11}-10^{-12} \mathrm{~s} \mathrm{~s}^{-1}$ for most sources. Such properties imply particularly strong surface dipole magnetic fields of the order of $10^{14}-10^{15} \mathrm{G}$. About 24 magnetars with these properties are known in our Galaxy, while one resides in the SMC and another in the LMC (Olausen \& Kaspi 2014). Most magnetars show high X-ray persistent luminosities, often surpassing their rotational energy reservoir, hence requiring an extra source of power. The latter is believed to be of magnetic origin, associated with their extremely strong outer or inner magnetic fields.

Magnetars are the most variable sources within the isolated NS zoo. Almost all have been observed to emit short $(\sim 0.1 \mathrm{~s})$, bright $\left(E_{\text {burst }} \approx 10^{37}-10^{41} \mathrm{erg}\right.$ ), hard X-ray bursts (see Mereghetti et al. 2015; Turolla et al. 2015, for reviews). Such bursting episodes can last days to weeks with varying numbers of bursts

\footnotetext{
${ }^{12}$ Deceased 2017 February 6.
}

emitted by a given source, ranging from tens to hundreds (e.g., Israel et al. 2008; Lin et al. 2011; van der Horst et al. 2012). These bursting episodes are usually accompanied by changes in the source persistent X-ray emission; an increase by a factor of a few to $\sim 100$ in flux level is usually observed to follow bursting episodes, together with a hardening in the X-ray spectrum (e.g., Esposito et al. 2011; Ng et al. 2011; Kaspi et al. 2014; Coti Zelati et al. 2015). Both properties usually relax quasi-exponentially to preburst levels on timescales of weeks to months (Rea \& Esposito 2011). Their pulse properties also vary following bursting episodes, with a change in shape and pulse fraction (e.g., Göğüş et al. 2002; Woods et al. 2004, see Woods \& Thompson 2006; Mereghetti 2008 for a review). We note that the magnetar-defining observational characteristics mentioned above have also been observed recently from NSs not originally classified as magnetars, like the high-B pulsars PSR J1846-0258 (Gavriil et al. 2008) and PSR J1119-6127 (Archibald et al. 2016; Göğuss et al. 2016), the central compact object in RCW 103 (Rea et al. 2016), and a low-B magnetar, SGR J0418+5729 (Rea et al. 2013; see also Scholz et al. 2014; Zhou et al. 2014). Moreover, a surrounding wind nebula, usually a pulsar-associated 
phenomenon, has now been observed from at least one magnetar, Swift J1834.9-0846 (Younes et al. 2012, 2016; Granot et al. 2017; Torres 2017).

Some magnetars also show bright, hard X-ray emission $(>10 \mathrm{keV})$ with total energy occasionally exceeding that of their soft X-ray emission. This hard emission is nonthermal in origin, phenomenologically described as a power law (PL) with a photon index in the range $\Gamma \sim 1-2$ (see, e.g., Kuiper et al. 2006). The hard and soft component properties may also differ (e.g., An et al. 2013; Vogel et al. 2014; Tendulkar et al. 2015). In the context of the magnetar model, the hard $\mathrm{X}$-ray emission has been explained as resonant Compton scattering of the soft (surface) emission by plasma in the magnetosphere (Baring \& Harding 2007; Fernández \& Thompson 2007; Beloborodov 2013).

So far, only four magnetars have been detected to show pulsed radio emission, excluding the high-B pulsar PSR J1119-6127 that exhibited magnetar-like activity (Weltevrede et al. 2011; Antonopoulou et al. 2015; Archibald et al. 2016; Göğüş et al. 2016). The radio emission from the four typical magnetars showed transient behavior, correlated with the X-ray outburst onset (Camilo et al. 2006, 2007; Levin et al. 2010). Rea et al. (2012) showed that all radio magnetars have $L_{\mathrm{X}} / \dot{E}<1$ during quiescence. However, the physical mechanism for the radio emission in magnetars (as well as why it has only been detected in a very small number of sources) remains largely unclear (e.g., Szary et al. 2015) and could be inhibited if optimal conditions for the production of pairs are not present (e.g., Baring \& Harding 1998).

SGR J1935+2154 is a recent addition to the magnetar family, discovered with the Swift/X-Ray Telescope (XRT) on 2014 July 05 (Stamatikos et al. 2014). Subsequent Swift, Chandra and $X M M$-Newton observations taken in 2014 confirmed the source as a magnetar with a spin period $P=3.25 \mathrm{~s}$ and $\dot{P}=1.43 \times$ $10^{-11} \mathrm{~s} \mathrm{~s}^{-1}$, implying a surface dipole B field of $B=2.2 \times 10^{14}$ $\mathrm{G}$ (Israel et al. 2016b). SGR J1935+2154 has been quite active since its discovery with at least three other outbursts: 2015 February 22, 2016 May 14, and 2016 June 18. The source is close to the geometrical center of the supernova remnant G57.2 + 0.8 (Gaensler 2014). The distance to the source is unknown, so we adopt a nominal distance to the magnetar of $9 \mathrm{kpc}$ for consistency with Israel et al. (2016b).

In this paper, we report on the analysis of all X-ray observations of SGR J1935+2154 taken after 2014 May, including a Nuclear Spectroscopic Telescope Array (NuSTAR) observation made within days of the 2015 outburst identifying the broadband X-ray spectrum of the source. We also report on the analysis of radio observations taken with Arecibo following the 2015 and 2016 June outbursts. X-ray and radio observations and data reduction are reported in Section 2, and analysis results are shown in Section 3. Section 4 discusses the results in the context of the magnetar model, while Section 5 summarizes our findings.

\section{Observations and Data Reduction}

\subsection{Chandra}

Chandra observed SGR J1935+2154 three times during its 2014 outburst and once during its 2016 June outburst. Two of the 2014 observations were in continuous-clocking (CC) mode, while the other two were taken in timed-exposure (TE) mode with one-eighth subarray. We analyzed these observations using CIAO 4.8.2 and calibration files CALDB version 4.7.2.
For the TE-mode observations, we extracted source events from a circle with radius $2^{\prime \prime}$, while background events were extracted from an annulus centered on the source with inner and outer radii of $4^{\prime \prime}$ and $10^{\prime \prime}$, respectively. Source events from the CC-mode observations were extracted using a box extraction region of $4^{\prime \prime}$ length. Background events were extracted from two box regions with the same length on each side of the source region. We used the CIAO specextract ${ }^{13}$ script to extract source and background spectral files, including response RMF and ancillary ARF files. Finally, we grouped the spectra to have only five counts per bin. Table 1 lists the details of the Chandra observations.

\subsection{XMM-Newton}

We analyzed all of the 2014 XMM-Newton observations of SGR J1935+2154. In all cases, the EPIC-pn (Strüder et al. 2001) camera was operated in Full Frame mode. The MOS cameras, on the other hand, were operated in small window mode. Both cameras used the medium filter. All data products were obtained from the XMM-Newton Science Archive $(\mathrm{XSA})^{14}$ and reduced using the Science Analysis System (SAS) version 14.0.0.

The PN and MOS data were selected using event patterns 0-4 and 0-12, respectively, and excluding X-ray events at the edge of the CCD or falling near a hot pixel ("FLAG $=0$ "). We inspected all observations for intervals of high background, such as due to solar flares, and excluded those where the background level was above $5 \%$ of the source flux. The source $\mathrm{X}$-ray flux was never high enough to cause pileup.

Source events for all observations were extracted from a circle with center and radius obtained by running the task eregionanalyse on the cleaned event files. This task calculates the optimum centroid of the count distribution within a given source region and the radius of a circular extraction region that maximizes the source signal-to-noise ratio $(\mathrm{S} / \mathrm{N})$. The radii of these extraction regions ranged from $40^{\prime \prime}$ to $50^{\prime \prime}$. Background events were extracted from a source-free annulus centered at the source with inner and outer radii of $60^{\prime \prime}$ and $100^{\prime \prime}$, respectively. We generated response matrix files using the SAS task rmfgen, while ancillary response files were generated using the SAS task arfgen. Again, we grouped the spectra to have only five counts per bin. Table 1 lists the details of the XMMNewton observations.

\subsection{Swift}

We reduced all 2014, 2015, and 2016 Swift/XRT (Burrows et al. 2005) data using xrtpipeline version 13.2, and we performed the analysis using HEASOFT version 6.20. The count rate for all but two Swift/XRT observations was between 0.01 and 0.05 counts $\mathrm{s}^{-1}$, so as a balance between minimizing the background and maximizing the $\mathrm{S} / \mathrm{N}$, we extracted source events from a $30^{\prime \prime}$ radius circle centered on the source (Evans et al. 2007, 2009). For obs IDs 00686761000 (0.057 counts s $\left.{ }^{-1}\right)$ and $00701182000\left(0.088\right.$ counts s$\left.^{-1}\right)$, we used a circular extraction region with a 47" radius (Evans et al. 2007, 2009). Background events were extracted from an annulus centered at the same position as the source with inner and outer radii of $80^{\prime \prime}$ and $120^{\prime \prime}$, respectively. Finally, we generated the ancillary files

\footnotetext{
13 http://cxc.harvard.edu/ciao/ahelp/specextract.html

14 http://xmm.esac.esa.int/xsa/index.shtml
} 
Table 1

Log of X-Ray Observations

\begin{tabular}{|c|c|c|}
\hline Telescope/Obs. ID & $\begin{array}{l}\text { Date } \\
\text { (MJD) }\end{array}$ & $\begin{array}{c}\text { Net Exposure } \\
(\mathrm{ks})\end{array}$ \\
\hline \multicolumn{3}{|c|}{2014} \\
\hline Swift-XRT/00603488000 & 56843.40 & 3.37 \\
\hline Swift-XRT/00603488001 & 56843.52 & 9.90 \\
\hline Swift-XRT/00603488003 & 56845.25 & 3.93 \\
\hline Swift-XRT/00603488004 & 56845.98 & 9.31 \\
\hline Swift-XRT/00603488006 & 56846.66 & 3.67 \\
\hline Swift-XRT/00603488007 & 56847.60 & 3.63 \\
\hline Swift-XRT/00603488008 & 56851.52 & 5.33 \\
\hline Swift-XRT/00603488009 & 56851.32 & 2.95 \\
\hline Chandra/15874 & 56853.59 & 9.13 \\
\hline Swift-XRT/00603488011 & 56858.00 & 2.95 \\
\hline Chandra/15875 & 56866.03 & 75.1 \\
\hline Chandra/17314 & 56900.03 & 29.0 \\
\hline XMM-Newton $/ 0722412501$ & 56926.95 & 16.9 \\
\hline XMM-Newton $/ 0722412601$ & 56928.20 & 17.8 \\
\hline XMM-Newton/0722412701 & 56934.36 & 16.1 \\
\hline XMM-Newton $/ 0722412801$ & 56946.11 & 8.61 \\
\hline XMM-Newton $/ 0722412901$ & 56954.15 & 6.53 \\
\hline XMM-Newton/0722413001 & 56957.95 & 12.4 \\
\hline XMM-Newton $/ 0748390801$ & 56976.16 & 9.83 \\
\hline \multicolumn{3}{|c|}{2015} \\
\hline Swift-XRT/00632158000 & 57075.51 & 7.33 \\
\hline Swift-XRT/00632158001 & 57075.80 & 1.80 \\
\hline Swift-XRT/00632158002 & 57076.52 & 5.91 \\
\hline Swift-XRT/00033349014 & 57078.18 & 3.13 \\
\hline NuSTAR/90001004002 & 57080.22 & 50.6 \\
\hline Swift-XRT/00033349015 & 57080.24 & 5.94 \\
\hline Swift-XRT/00033349016 & 57085.31 & 3.94 \\
\hline Swift-XRT/00033349017 & 57092.55 & 3.91 \\
\hline Swift-XRT/00033349018 & 57102.00 & 4.37 \\
\hline Swift-XRT/00033349019 & 57127.16 & 1.97 \\
\hline Swift-XRT/00033349020 & 57127.77 & 2.94 \\
\hline Swift-XRT/00033349021 & 57128.56 & 2.66 \\
\hline Swift-XRT/00033349022 & 57129.10 & 0.85 \\
\hline Swift-XRT/00033349023 & 57134.35 & 1.37 \\
\hline Swift-XRT/00033349024 & 57220.96 & 1.98 \\
\hline Swift-XRT/00033349025 & 57377.70 & 3.94 \\
\hline \multicolumn{3}{|c|}{2016} \\
\hline Swift-XRT/00686761000 & 57526.38 & 1.67 \\
\hline Swift-XRT/00686842000 & 57527.24 & 0.84 \\
\hline Swift-XRT/00033349026 & 57527.77 & 2.96 \\
\hline Swift-XRT $/ 00687123000^{\mathrm{a}}$ & 57529.84 & 1.21 \\
\hline Swift-XRT/00687124000 & 57529.85 & 0.81 \\
\hline Swift-XRT/00033349028 & 57539.87 & 2.78 \\
\hline Swift-XRT/00033349029 & 57540.54 & 0.47 \\
\hline Swift-XRT/00033349031 & 57554.16 & 2.57 \\
\hline Swift-XRT/00033349032 & 57561.02 & 1.58 \\
\hline Swift-XRT/00701182000 & 57562.81 & 1.65 \\
\hline Swift-XRT/00701590000 & 57565.58 & 1.39 \\
\hline Swift-XRT/00033349033 & 57567.18 & 2.01 \\
\hline Swift-XRT/00033349034 & 57569.52 & 2.38 \\
\hline Chandra/18884 & 57576.23 & 18.2 \\
\hline Swift-XRT/00033349035 & 57576.77 & 2.78 \\
\hline Swift-XRT/00033349036 & 57586.20 & 2.48 \\
\hline Swift-XRT/00033349037 & 57597.04 & 2.84 \\
\hline
\end{tabular}

Note.

${ }^{\mathrm{a}}$ Merged Swift exposures. with xrtmkarf and used the response matrices in CALDB v014. All spectra and ancillary files are corrected for the pointspread function (PSF) and exposure map corresponding to each observation. The log of the XRT observations is listed in Table 1.

All observations that resulted in a source number of counts $>30$ were included in the analysis individually. Observations with source number counts $<30$ were merged with other observations that were taken within a two-day interval. Any individual or merged observation that did not satisfy the 30 source number counts limit were excluded from the analysis. However, most of these lost intervals were compensated for with existing quasisimultaneous Chandra and XMM-Newton observations.

\subsection{NUSTAR}

The NUSTAR (Harrison et al. 2013) consists of two similar focal-plane modules (FPMA and FPMB) operating in the energy range $3-79 \mathrm{keV}$. It is the first hard X-ray $(>10 \mathrm{keV})$ focusing telescope in orbit.

NUSTAR observed SGR J1935+2154 on 2015 February 27 at 05:16:20 UTC. The net exposure time of the observation is $50.6 \mathrm{ks}$ (Table 1). We processed the data using the NuSTAR Data Analysis Software, nustardas version v1.5.1. We analyzed the data using the nuproducts task (which allows for spectral extraction and generation of ancillary and response files) and HEASOFT version 6.20. We extracted source events around the source position using a circular region with $40^{\prime \prime}$ radius. Background events were extracted from an annulus around the source position with inner and outer radii of $80^{\prime \prime}$ and $160^{\prime \prime}$, respectively.

\subsection{Arecibo Observations}

We observed SGR J1935+2154 with the $305 \mathrm{~m}$ William E. Gordon Telescope at the Arecibo Observatory in Puerto Rico, as part of Director's Discretionary Time, to search for radio emission after its X-ray activation, both in 2015 and in 2016. The source was observed on 2015 March 5, March 12, and March 27 (henceforth Obs. 1-3) and on 2016 July 5, July 12, and July 27 (Obs. 4-6). Observation durations ranged from $\sim 1$ to $2.5 \mathrm{hr}$; in each session (with the exception of Obs. 2 and 5), the observation time was split between two different observing frequencies. A short summary of all observations is presented in Table 2.

Observations using the Arecibo C-band receiver were performed at a central frequency of $4.6 \mathrm{GHz}$, using the seven Mock Spectrometers as backends. We used a bandwidth of $\sim 172 \mathrm{MHz}$ per Mock, each of which was split across 32 channels. The 7 Mock data sets were analyzed separately in order to avoid complications due to scintillation or radio frequency interference (RFI), which corrupted some bands much more heavily than others. Together the 7 Mocks spanned 4.1-5.2 GHz, with $\sim 22 \mathrm{MHz}$ overlap between the bands. The time resolution was $65 \mu$ s with 16-bit samples. In every C-band observation, we used the nearby and bright PSR B1919+21 to test the instrumental setup.

The Arecibo L-band Wide receiver was used in the frequency range $0.98-1.78 \mathrm{GHz}$ with a central frequency of $1.38 \mathrm{GHz}$. As back end, we used the Puerto-Rican Ultimate Pulsar Processing Instrument (PUPPI). PUPPI provided $800 \mathrm{MHz}$ bandwidth (roughly $500 \mathrm{MHz}$ usable after removing RFI and the edges of the receiver band), split across 2048 spectral channels. For our observations, PUPPI was used in 
Table 2

Arecibo Observations Summary

\begin{tabular}{|c|c|c|c|}
\hline Obs. & Project ID & Obs. Start Date & Integration Time (hr) \\
\hline \multicolumn{4}{|c|}{ C-band Observations } \\
\hline \multicolumn{4}{|c|}{$G=8 \mathrm{~K} \mathrm{Jy}^{-1}, T_{\mathrm{sys}}=28 \mathrm{~K}$} \\
\hline 1 & p2976 & 2015 Mar 05 & 1.0 \\
\hline 2 & p2976 & 2015 Mar 12 & 1.0 \\
\hline 3 & p2976 & 2015 Mar 27 & 1.3 \\
\hline 4 & p3100 & 2016 Jul 05 & 0.7 \\
\hline 5 & p3100 & 2016 Jul 12 & 1.0 \\
\hline 6 & p3100 & 2016 Jul 27 & 0.3 \\
\hline \multicolumn{4}{|c|}{ L-band Observations } \\
\hline \multicolumn{4}{|c|}{$G=10 \mathrm{~K} \mathrm{Jy}^{-1}, T_{\text {sys }}=33 \mathrm{~K}$} \\
\hline 1 & p2976 & 2015 Mar 05 & 1.0 \\
\hline 2 & p2976 & 2015 Mar 12 & $\cdots$ \\
\hline 3 & p2976 & 2015 Mar 27 & 1.0 \\
\hline 4 & p3100 & 2016 Jul 05 & 0.5 \\
\hline 5 & p3100 & 2016 Jul 12 & $\cdots$ \\
\hline 6 & p3100 & 2016 Jul 27 & 0.4 \\
\hline
\end{tabular}

Incoherent Search mode. The data were sampled at $40.96 \mu \mathrm{s}$ with eight bits per sample. At the start of every L-band observation, PSR J1924+1631 was observed to verify the setup.

\section{Results}

\subsection{X-Ray Imaging}

To assess the presence of any extended emission around SGR J1935+2154, we relied on the four Chandra observations, as well as the 2014 XMM-Newton observations.

Two of the Chandra observations, including the one in 2016 , were taken in TE mode, while the other two were taken in CC mode. For the two TE-mode observations, we simulated a Chandra PSF at the source position with the spectrum of SGR J1935+2154, using the Chandra ray trace $\left(\right.$ ChaRT $\left.^{15}\right)$ and MARX. ${ }^{16}$ The middle panel of Figure 1 shows the radial profile, in the energy range $0.8-8 \mathrm{keV}$, of the 2016 TE-mode observations, which had an exposure twice as long as the one taken in 2014. Black dots represent the radial profile of the actual observation, while the red squares represent the radial profile of the simulated PSF. There is no evidence for smallscale extended emission beyond a point source PSF in this observation. The 2014 observation showed similar results (G. Israel et al. 2017, private communication).

The CC-mode observations are not straightforward to perform imaging analysis with, given their 1D nature. To mitigate this limitation, we calculated and averaged the total number counts, detected in the energy range $0.8-8 \mathrm{keV}$, in each two pixels at equal distance from the central brightest pixel, up to a distance of $20^{\prime \prime}$ (we also split the central brightest pixel into two, to better sample the inner 0 ." 5 ). The background for these observations was estimated by averaging the number of counts from all pixels at a distance $25^{\prime \prime}-50^{\prime \prime}$ from both sides of the central brightest pixel. The left panel of Figure 1 shows the results of our analysis

\footnotetext{
15 http://cxc.harvard.edu/ciao/PSFs/chart2/

16 http://space.mit.edu/CXC/MARX/
}

on the longest of the two CC-mode observations, obs. ID 15875 (notice the $y$-axis units of counts $\operatorname{arcsec}^{-1}$ ). The solid horizontal line represents the background level, while the dots represent the 1D radial profile of SGR J1935+2154. The inset is a zoom-in at the $3^{\prime \prime}-20^{\prime \prime}$ region. The level of emission beyond $\sim 5^{\prime \prime}$ from the central pixel is consistent with the background, so we conclude that there is no evidence for small-scale extended emission from the source. We verified our results by converting our 2016 TEmode observation into $\mathrm{CC}$ mode by collapsing the counts into 1D. We then performed the same analysis on this converted image as the one done on the $\mathrm{CC}$-mode observations. The results are shown in the right panel of Figure $1 .^{17}$

XMM-Newton observations showed a weak extended emission, in the energy range $0.8-10 \mathrm{keV}$, after stacking all seven 2014 observations, in accordance with the results reported by Israel et al. (2016b).

\subsection{Timing \\ 3.2.1. X-Ray}

We searched the 2015 NUSTAR and 2016 Chandra data for the pulse period from SGR J1935+2154. We focused the search in an interval around the expected pulse period from the source at the NuSTAR and Chandra MJDs, after extrapolating the timing solution detected with Chandra and XMMNewton during the 2014 outburst (Israel et al. 2016b). We included the possibility of timing noise or glitches and searched an interval with $\delta \dot{P} \approx 1.5 \times 10^{-9} \mathrm{~s} \mathrm{~s}^{-1}$. We searched for a total of 960 independent frequencies in the frequency range $0.3030-0.3125 \mathrm{~Hz}$. For the FPMA and FPMB modules, we extracted events from a circle with a $45^{\prime \prime}$ radius around the source position and in the energy range $3-50 \mathrm{keV}$. We extracted the Chandra events using a $2^{\prime \prime}$ circle centered at the source in the energy range $1-8 \mathrm{keV}$. We barycenter-corrected the photon arrival times to the solar system barycenter.

We first applied the $Z_{m}^{2}$ test algorithm (Buccheri et al. 1983) at the NUSTAR data, where $m$ is the number of harmonics. Although the signal during the 2014 outburst was nearly sinusoidal, we applied the test using $m=1,2,3$, and 5, considering the possibility of a change in the pulse shape during the later outbursts. The highest peak, found in the $Z_{1}^{2}$ test in the NuSTAR data, with a significance of $3.2 \sigma$, corrected for trial frequencies and number of harmonics searched, is located at the period reported in Younes et al. (2015a) of 3.24729(1) s. This is largely different from the pulse period of 3.24528(6) s derived by Israel et al. (2016b) for the 2015 XMM-Newton observation taken a month later. The change in frequency between the two observations is about $1.2 \times 10^{-9} \mathrm{~s} \mathrm{~s}^{-1}$, too large to correspond to any timing noise. We also repeated our above analysis for different energy cuts, namely $3-10 \mathrm{keV}$ and $3-30 \mathrm{keV}$, and for different circular extraction regions of $30^{\prime \prime}$ and $37^{\prime \prime}$ radii (to optimize $\mathrm{S} / \mathrm{N}$ ). We find no other significant peaks in the $Z^{2}$ power for any of the above combinations. We, therefore, conclude that we do not detect the spin period of the source in our 2015 NuSTAR observation. Following the same method, we searched for the pulse period in the 2016 Chandra observation. Similarly, we do not detect the pulse period from SGR J1935+2154.

We estimated upper limits on the rms pulsed fraction (PF) of a pure sinusoidal modulation by simulating 10,000 light curves

\footnotetext{
17 We note that these results have been confirmed independently by G. Israel et al. 2017, private communication, and the referee.
} 

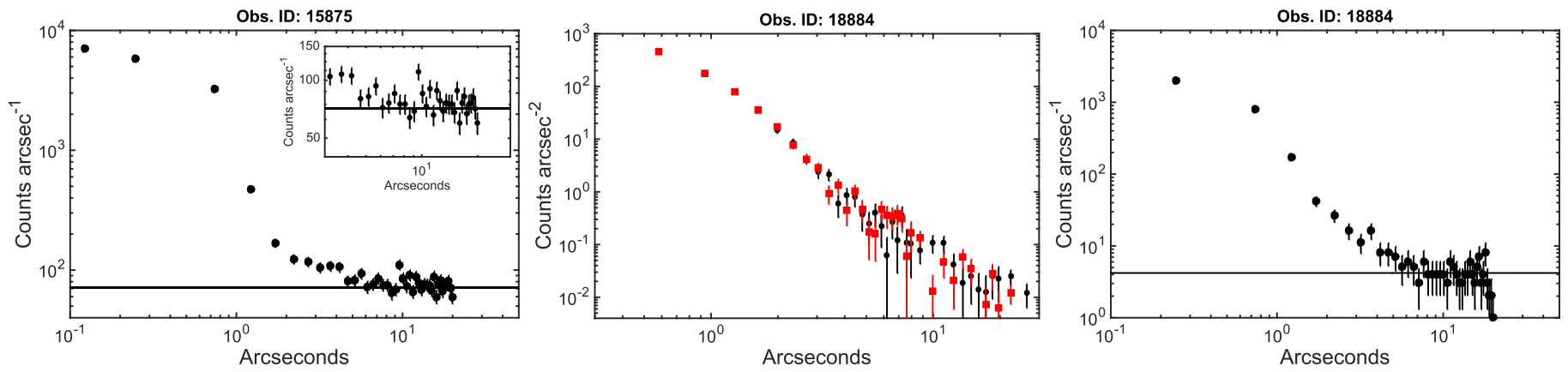

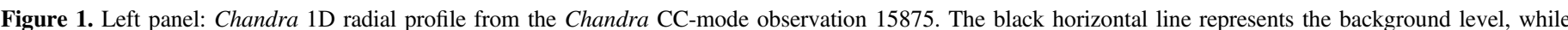

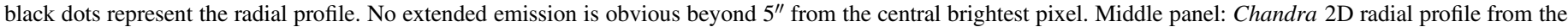

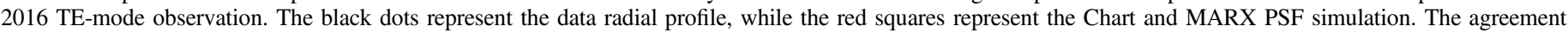

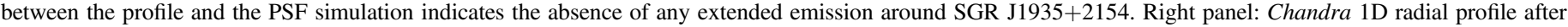

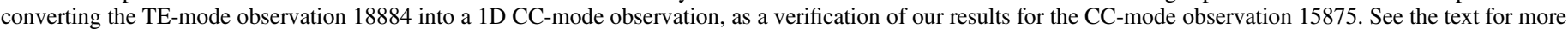
details.

with mean count rate corresponding to the true backgroundcorrected count rate of the source and pulsed at a given rms PF. For our $2015 \mathrm{NuSTAR}$ observation, we derive a $3 \sigma(99.73 \%$ confidence) upper limit on the rms PF of $26 \%, 35 \%$, and $43 \%$ in the energy ranges $3-50 \mathrm{keV}, 3-10 \mathrm{keV}$, and $10-50 \mathrm{keV}$, respectively. For our 2016 Chandra observation, we set a $3 \sigma$ rms PF upper limit of $8 \%$. These limits are consistent with the $5 \%$ rms pulsed fraction derived during the 2015 XMM-Newton observation in the $0.5-10 \mathrm{keV}$ range (Israel et al. 2016b).

\subsubsection{Radio}

A consistent method was adopted for both the C-band and L-band data analyses and was based on tools from the pulsar search and analysis software PRESTO (Ransom 2001; Ransom et al. 2002, 2003). To excise RFI, we created a mask using rfifind. After RFI excision, we used three different techniques to search for radio pulsations: (1) a blind, Fourier-based periodicity search, (2) a search based on the known spin parameters from an X-ray-derived ephemeris (described below), and (3) a single-pulse search to look for sporadic pulses from the magnetar.

Blind searches. We conducted coherent pulsation searches using no a priori assumption about the spin period in order to allow for a change compared to the ephemeris (e.g., a glitch) or the serendipitous discovery of a pulsar in the field. Using prepsubband, we created barycentered and RFI-excised time series for a DM range of $0-2160 \mathrm{pc} \mathrm{cm}^{-3}$, where the trial DM spacing was determined using DDplan. We then Fourier transformed each time series with realfft and conducted accelsearch-based searches (with a maximum signal drift of $z_{\max }=100$ for the $\mathrm{C}$ band and $z_{\max }=10$ for the $\mathrm{L}$ band in the power spectrum) in order to maintain sensitivity to a possible binary orbit. The most promising candidates from this search were collated and ranked using ACCEL_sift. We folded the raw filterbank data for the best 200 candidates identified with ACCEL_sift and then visually inspected each candidate signal using parameters such as cumulative $\mathrm{S} / \mathrm{N}$, $\mathrm{S} / \mathrm{N}$ as a function of DM, pulse profile shape, and broadbandedness as deciding factors in judging whether a certain candidate was plausibly of astrophysical origin or whether it was likely to be noise or RFI. We found no plausible astrophysical signals in this analysis.

Ephemeris-based searches. Coherent X-ray pulsations from SGR J1935+2154 were detected by Israel et al. (2014) at a
$>10 \sigma$ confidence level. Upon this discovery, SGR J1935 +2154 was monitored using XMM-Newton and Chandra observations between 2014 and 2015 (see Israel et al. 2016a). This campaign resulted in a timing solution as presented in Table 2 of Israel et al. (2016a). We used the period, period derivative, and second period derivative from this ephemeris to extrapolate the source spin period for Obs. 1-3 and Obs. 4-6 (average $3.2452373 \mathrm{~s}$ and $3.2453063 \mathrm{~s}$, respectively). We then folded the raw data from each C-band and L-band observation with the appropriate spin period using PRESTO's prepfold. This folding operation was restricted to only optimize $\mathrm{S} / \mathrm{N}$ over a small search range in pulse period and DM and incorporated the RFI mask. We repeated this folding routine over dispersion measures (DMs) ranging from 0 to $1000 \mathrm{pc} \mathrm{cm}^{-3}$ in steps of $50 \mathrm{pc} \mathrm{cm}^{-3}$. A similar ephemeris-based folding operation was carried out over the dedispersed time series generated in the above "blind search." Here, our folding operation involved spin periods computed for each observation, and we prohibited prepfold from searching over spin-period derivative. We obtained 46,930 folded candidates for the $\mathrm{L}$ band and 6120 candidates for the $\mathrm{C}$ band. Inspection of these candidates based on pulse profile and $\mathrm{S} / \mathrm{N}$ returned no promising candidates.

Recently, using the intermediate flare from SGR J1935 +2154 along with a magnetic field estimate from the timing analysis of Israel et al. (2016a) and Kozlova et al. (2016) showed that the magnetar is at a distance of $<10 \mathrm{kpc}$. We used the NE2001 Galactic electron density model and integrated in the source direction up to $10 \mathrm{kpc}$ to obtain an expected DM. We obtain a value of $344 \mathrm{pc} \mathrm{cm}^{-3}$ (typical error is $20 \%$ fractional), which lies well within the DM range of our coherent pulsation searches. These searches found no plausible radio pulsations from SGR J1935+2154.

We estimate maximum flux density limits using the radiometer equation (see Dewey et al. 1985; Bhattacharya 1998; Lorimer \& Kramer 2012) given by

$$
S_{\min }=\frac{\left(\frac{S}{N}\right) \beta T_{\mathrm{sys}}}{G \sqrt{n_{p} t_{\mathrm{obs}} \triangle f}} \sqrt{\frac{W}{P-W}},
$$

where $S_{\min }$ is the minimal detectable flux density (mJy), $G$ is the gain of the telescope $\left(\mathrm{K} \mathrm{Jy}^{-1}\right), \beta$ is a correction factor that is $\sim 1$ for a large number of bits per sample, $T_{\text {sys }}$ is the system noise temperature $(\mathrm{K}), \Delta f$ is the bandwidth $(\mathrm{MHz})$, and $t_{\mathrm{obs}}$ is the integration time (s) for a given source. These parameters for 
the observational setup in each band are listed in Table 2. We assume a pulsar duty cycle $(W / P)$ of $20 \%$ and a minimum detectable $\mathrm{S} / \mathrm{N}$ of 10 in our search. This yields a maximum (average) flux density limit of $14 \mu \mathrm{Jy}$ for the C-band observations and $7 \mu \mathrm{Jy}$ for the L-band observations.

Single-pulse search. The previously described pulsation searches focused on finding persistent radio pulsations. However, it is also possible that SGR J1935+2154 emits sporadic individual radio pulses. Therefore, we searched for such pulses using a matched filtering analysis with boxcar functions of widths $65 \mu \mathrm{s}$ to $\sim 100 \mathrm{~ms}$ (depending somewhat on the trial DM), as implemented in PRESTO's single_pulse_search.py routine. We inspected the resulting candidates by looking for events that peaked in signal-to-noise ratio at specific DMs. All detected signals were consistent with being due to RFI, including one intrinsically swept-frequency emitter with harmonically related $\mathrm{S} / \mathrm{N}$ peaks at DMs $\sim 250$, 500 , and $1000 \mathrm{pc} \mathrm{cm}^{-3}$. We thus find no conclusive evidence for sporadic individual radio pulses from SGR J1935+2154, down to a peak flux density limit of $\sim 10 / 20 \mathrm{mJy}$ (L-band/ C-band) for a fiducial $10 \mathrm{~ms}$ pulse detected at $10 \sigma$.

\subsection{X-Ray Spectroscopy}

We fit our spectra in the energy range $0.8-8 \mathrm{keV}$ for Chandra, 0.8-10 keV for XMM-Newton and Swift (emission is background dominated at energies $<0.8 \mathrm{keV}$ ), and $3-50 \mathrm{keV}$ for NUSTAR, using XSPEC (Arnaud 1996) version 12.9.0k. We used the photoelectric cross sections of Verner et al. (1996) and the abundances of Wilms et al. (2000) to account for absorption by neutral gas. For all spectral fits using different instruments, we added a multiplicative constant normalization, frozen to one for the spectrum with the highest $\mathrm{S} / \mathrm{N}$ and allowed to vary for the other instruments. This takes into account any calibration uncertainties between the different instruments. We find that this uncertainty is between $2 \%$ and $8 \%$. For all spectral fitting, we used the Cash statistic (C-stat) in XSPEC for model parameter estimation and error calculation, while the goodness command was used for goodness-of-fit estimation. We note that the C-stat implementation in XSPEC allows for a background to be read in instead of modeled. Nevertheless, we verified our results for the XMM-Newton and NUSTAR spectra, which have a larger background than Chandra and Swift, using the typical $\chi^{2}$ method after binning the spectra to have an $\mathrm{S} / \mathrm{N}$ of 4.5. We find consistent results between the C-stat and $\chi^{2}$ methods. All quoted uncertainties are at the $1 \sigma$ level, unless otherwise noted.

\subsubsection{The 2014 Outburst}

We started our spectral analysis of the 2014 outburst (Table 1) by focusing on the high $\mathrm{S} / \mathrm{N}$ spectra derived from the seven XMM-Newton observations (PN+MOS1+MOS2). First, we fit these spectra simultaneously with an absorbed (tbabs in XSPEC) blackbody plus power-law (BB+PL) model, allowing all spectral model parameters to vary freely, that is, BB temperature (kT) and emitting area, and PL photon index $(\Gamma)$ and normalization, except for the absorption hydrogen column density, which we linked between all spectra. This model provides a good fit to the data with a C-stat of 5116.7 for 5196 degrees of freedom (d.o.f.). We find a hydrogen column density $N_{\mathrm{H}}=(2.4 \pm 0.1) \times 10^{22} \mathrm{~cm}^{-2}$. The $\mathrm{BB}$ temperatures and PL indices are consistent for all spectra within the $1 \sigma$ level. Hence, we linked these parameters and refit. We find a C-stat of 5131.75 for 5208 d.o.f. To estimate which model is preferred by the data (here and elsewhere in the text), we estimate the difference in the Bayesian information criterion (BIC), where a $\triangle \mathrm{BIC}$ of 8 is considered significant, and the model with the lower BIC is preferred (e.g., Liddle 2007). Comparing the case of free versus linked $k T$ and $\Gamma$, we find that the case of linked parameters is preferred with a $\triangle \mathrm{BIC} \approx 88$. This fit resulted in a hydrogen column density $N_{\mathrm{H}}=2.4 \pm 0.1 \times 10^{22} \mathrm{~cm}^{-2}$, a BB temperature $k T=0.46 \pm 0.01 \mathrm{keV}$ and area $R=1.45_{-0.03}^{+0.07} \mathrm{~km}$, and a photon index $\Gamma=2.0_{-0.5}^{+0.4}$.

We then fit the three Chandra spectra simultaneously, linking the hydrogen column density, while leaving all other fit parameters free to vary. We find a common hydrogen column density $N_{\mathrm{H}}=(2.9 \pm 0.3) \times 10^{22} \mathrm{~cm}^{-2}$. Similar to the case of the XMM-Newton observations, the $\mathrm{BB}$ temperature and the $\mathrm{PL}$ photon index were consistent within the $1 \sigma$ confidence level among the three observations. We, therefore, linked the $\mathrm{BB}$ temperature and the PL photon index in the three observations and found $k T=0.46 \pm 0.02, R=1.8 \pm 0.2 \mathrm{~km}$, and $\Gamma=2.4_{-0.6}^{+0.4}$.

Given the consistency in $N_{\mathrm{H}}, \mathrm{BB}$ temperature, and $\mathrm{PL}$ photon index between the Chandra and XMM-Newton observations, we then fit the spectra from all 10 observations simultaneously, first only linking the $N_{\mathrm{H}}$ among all observations. We find a good fit with a C-stat of 5750 for 5845 d.o.f, with $N=(2.4 \pm 0.1) \times 10^{22} \mathrm{~cm}^{-2}$. Similar to the above two cases, we find that the BB temperatures and the PL indices are consistent within $1 \sigma$. Hence, we fit all 10 observations while linking $k T$ and $\Gamma$. We find a C-stat of 5806 for 5863 d.o.f. Comparing this fit to the above case, we find a $\triangle \mathrm{BIC}=100$, suggesting that the latter fit is preferred over the fit where parameters were left free to vary. The best-fit spectral parameters for the $\mathrm{BB}+\mathrm{PL}$ model are summarized in Table 3, while the data and best-fit model are shown in Figure 2.

We also fit all spectra with an absorbed $\mathrm{BB}+\mathrm{BB}$ model following the above methodology. We first only link $N_{\mathrm{H}}$ among all spectra while allowing the temperature and emitting area of the two BBs free to vary. We find that the BB temperature of the cool component as well as the hot component are consistent at the $1 \sigma$ level among all 10 observations and were, therefore, linked. This alternative fit resulted in a C-stat of 5812 for 5863 d.o.f., similar in goodness to the BB+PL fit. Table 3 gives the $\mathrm{BB}+\mathrm{BB}$ best-fit spectral parameters, while the data and best-fit model are shown in Figure 2.

We analyzed the Swift/XRT observations taken during the 2014 outbursts following the procedure explained in Section 2.3. We fit all XRT spectra simultaneously with the $\mathrm{BB}+\mathrm{PL}$ and $\mathrm{BB}+\mathrm{BB}$ models. Due to the limited statistics, we fixed the temperatures and the photon indices to the values derived with the above XMM-Newton + Chandra fits. We made sure that the resulting fit was statistically acceptable using the XSPEC goodness command. In the event of a statistically bad fit, we allowed the temperatures and the photon indices to vary within the $3 \sigma$ uncertainty of the XMM-Newton+Chandra fits, which did give a statistically acceptable fit in all cases. We show in Figure 3 the flux evolution of the BB+PL model and 
Table 3

Best-fit XMM-Newton and Chandra X-Ray Spectral Parameters

\begin{tabular}{|c|c|c|c|c|c|c|c|}
\hline Obs. ID & $10^{22} \mathrm{~cm}^{N_{\mathrm{H}}}$ & $\begin{array}{l}k T_{\text {cool }} \\
(\mathrm{keV})\end{array}$ & $\begin{array}{c}R_{\text {cool }} \mathrm{a}^{\mathrm{a}} \\
(\mathrm{km})\end{array}$ & $\begin{array}{l}\Gamma / k T_{\text {hot }} \\
(/ \mathrm{keV})\end{array}$ & $\begin{array}{c}R_{\mathrm{hot}}^{\mathrm{a}} \\
\left(10^{-3} \mathrm{~km}\right)\end{array}$ & $\begin{array}{l}F_{\mathrm{kT}-\text { cool }} \\
\left(10^{-12}, \mathrm{erg} \mathrm{s}^{-1} \mathrm{~cm}^{-2}\right)\end{array}$ & $\begin{array}{c}F_{\mathrm{PL} / \mathrm{kT}-\mathrm{hot}} \\
\left(10^{-12}, \mathrm{erg} \mathrm{s}^{-1} \mathrm{~cm}^{-2}\right)\end{array}$ \\
\hline \multicolumn{8}{|c|}{2014 Outburst-BB+PL-Goodness 54\% } \\
\hline 15874 & $2.46 \pm 0.08$ & $0.47 \pm 0.01$ & $1.7 \pm 0.08$ & $2.0 \pm 0.2$ & $\ldots$ & $1.78 \pm 0.16$ & $1.31 \pm 0.33$ \\
\hline 15875 & (L) & (L) & $1.8 \pm 0.05$ & (L) & $\ldots$ & $2.01 \pm 0.09$ & $1.27 \pm 0.27$ \\
\hline 17314 & (L) & (L) & $1.8 \pm 0.06$ & (L) & $\ldots$ & $1.96 \pm 0.08$ & $0.75 \pm 0.19$ \\
\hline 0722412501 & (L) & (L) & $1.6 \pm 0.05$ & (L) & $\ldots$ & $1.50 \pm 0.06$ & $0.69 \pm 0.17$ \\
\hline 0722412601 & (L) & (L) & $1.6 \pm 0.05$ & (L) & $\ldots$ & $1.49 \pm 0.06$ & $0.62 \pm 0.15$ \\
\hline 0722412701 & (L) & (L) & $1.6 \pm 0.05$ & (L) & $\ldots$ & $1.56 \pm 0.06$ & $0.64 \pm 0.16$ \\
\hline 0722412801 & (L) & (L) & $1.6 \pm 0.06$ & (L) & $\ldots$ & $1.57 \pm 0.07$ & $0.69 \pm 0.17$ \\
\hline 0722412901 & (L) & (L) & $1.6 \pm 0.06$ & (L) & $\ldots$ & $1.50 \pm 0.08$ & $0.65 \pm 0.17$ \\
\hline 0722413001 & (L) & (L) & $1.5 \pm 0.05$ & (L) & $\ldots$ & $1.42 \pm 0.07$ & $0.66 \pm 0.17$ \\
\hline 0748390801 & (L) & (L) & $1.5 \pm 0.05$ & (L) & $\ldots$ & $1.38 \pm 0.09$ & $0.90 \pm 0.21$ \\
\hline \multicolumn{8}{|c|}{2016 Outburst-Goodness $61 \%$} \\
\hline 18884 & $2.7 \pm 0.3$ & $0.42 \pm 0.04$ & $2.3 \pm 0.5$ & $1.3_{-0.7}^{+0.9}$ & $\ldots$ & $2.0 \pm 0.3$ & $1.1 \pm 0.6$ \\
\hline \multicolumn{8}{|c|}{2014 Outburst-BB+BB-Goodness 47\% } \\
\hline 15874 & $2.30 \pm 0.04$ & $0.48 \pm 0.01$ & $1.8 \pm 0.6$ & $1.6 \pm 0.1$ & $80 \pm 9$ & $2.12 \pm 0.09$ & $0.53 \pm 0.08$ \\
\hline 15875 & (L) & (L) & $1.9 \pm 0.6$ & (L) & $79 \pm 9$ & $2.34 \pm 0.05$ & $0.52 \pm 0.03$ \\
\hline 17314 & (L) & (L) & $1.8 \pm 0.6$ & (L) & $61 \pm 8$ & $2.10 \pm 0.06$ & $0.31 \pm 0.04$ \\
\hline 0722412501 & (L) & (L) & $1.6 \pm 0.5$ & (L) & $57 \pm 7$ & $1.66 \pm 0.04$ & $0.27 \pm 0.02$ \\
\hline 0722412601 & (L) & (L) & $1.5 \pm 0.6$ & (L) & $54 \pm 7$ & $1.62 \pm 0.04$ & $0.25 \pm 0.02$ \\
\hline 0722412701 & (L) & (L) & $1.6 \pm 0.5$ & (L) & $55 \pm 7$ & $1.70 \pm 0.04$ & $0.25 \pm 0.02$ \\
\hline 0722412801 & (L) & (L) & $1.6 \pm 0.5$ & (L) & $57 \pm 7$ & $1.72 \pm 0.05$ & $0.28 \pm 0.03$ \\
\hline 0722412901 & (L) & (L) & $1.6 \pm 0.6$ & (L) & $55 \pm 8$ & $1.65 \pm 0.05$ & $0.26 \pm 0.03$ \\
\hline 0722413001 & (L) & (L) & $1.5 \pm 0.6$ & (L) & $56 \pm 7$ & $1.57 \pm 0.04$ & $0.26 \pm 0.03$ \\
\hline 0748390801 & (L) & (L) & $1.5 \pm 0.6$ & (L) & $65 \pm 8$ & $1.62 \pm 0.05$ & $0.36 \pm 0.03$ \\
\hline \multicolumn{8}{|c|}{2016 Outburst-Goodness 55\% } \\
\hline 18884 & $2.7 \pm 0.3$ & $0.43 \pm 0.02$ & $2.3 \pm 0.4$ & $2.0_{-0.5}^{+1.3}$ & $52_{-26}^{+36}$ & $2.5 \pm 0.3$ & $0.45_{-0.06}^{-0.07}$ \\
\hline
\end{tabular}

Note. Fluxes are derived in the energy range $0.5-10 \mathrm{keV}$. All fluxes are corrected for absorption. (L) represents a linked parameter between the different spectra.

${ }^{a}$ Assuming a distance of $9 \mathrm{kpc}$.

in Figure 4 the area evolution of the 2BB model. These results are discussed in Section 4.

Finally, we note that during the 2015 outburst, which will be discussed in Section 3.3.2, NuSTAR reveals a hard X-ray component dominating the spectrum at energies $>10 \mathrm{keV}$ and with a nonnegligible contribution at energies $5-10 \mathrm{keV}$. In order to understand the effect of such a hard component on the spectral shape below $10 \mathrm{keV}$ (if it indeed exists during the 2014 outburst), we added a hard PL component to the two above models (i.e., BB+PL and 2BB) while fitting the seven XMMNewton observations. We fixed its index and normalization to the result of a PL fit to the $N u S T A R$ data from 10 to $50 \mathrm{keV}$. $^{18}$ As one would expect, we find that the addition of this extra hard PL results in a softening of the $<10 \mathrm{keV}$ PL and hot BB components. On average, we find a photon index for the soft PL $\Gamma=2.7 \pm 0.3$. For the $2 \mathrm{BB}$ model, we find a temperature for the hot $\mathrm{BB} k T=0.8 \pm 0.2$ with a radius for the emitting area $R \approx 210 \pm 30 \mathrm{~m}$. Moreover, we find the fluxes of the lowenergy $\mathrm{PL}$ or the hot $\mathrm{BB}$ to be a factor of $\sim 3$ lower; however, the total $0.5-10 \mathrm{keV}$ flux is similar to the above two models when we did not include a contribution from a hard PL. We cannot, unfortunately, add a hard PL component to the XRT

\footnotetext{
${ }^{18}$ We used a simultaneous Swift/XRT observation to properly normalize the flux of this hard PL component to the 2014 XMM-Newton ones, assuming that the PL flux below and above $10 \mathrm{keV}$ varies in tandem.
}

spectra and still extract meaningful flux values from the two other components $<10 \mathrm{keV}$, due to very limited statistics. A complete statistical analysis, invoking many spectral simulations, aiming at understanding the exact effect of a hard PL component on the spectral curvature $<10 \mathrm{keV}$, is beyond the scope of this paper. In all our discussions in Section 4, however, we made sure to avoid making any conclusions that could be affected by such a shortcoming of the data as we are considering here.

\subsubsection{The 2015 Outburst}

For the 2015 outburst, we first concentrated on the analysis of the simultaneous NuSTAR and Swift/XRT observations (Table 1) taken on February 27, five days following the outburst onset. This provided the first look at the broadband X-ray spectrum of the source. SGR J1935+2154 is clearly detected in the two NUSTAR modules with a backgroundcorrected number of counts of $\sim 800(3-50 \mathrm{keV})$. We find a background-corrected number of counts in the $3-10 \mathrm{keV}$ and $10-50 \mathrm{keV}$ ranges of about 500 and 300 counts, respectively. The simultaneous XRT observation provided about 130 background-corrected counts in the energy range $0.5-10 \mathrm{keV}$.

We then fit the spectra simultaneously to an absorbed $\mathrm{BB}+\mathrm{PL}$ model. We find a good fit with a C-stat of 444 for 452 d.o.f., with an $N_{\mathrm{H}}=\left(2.0_{-0.7}^{+0.8}\right) \times 10^{22} \mathrm{~cm}^{-2}$. We find a 

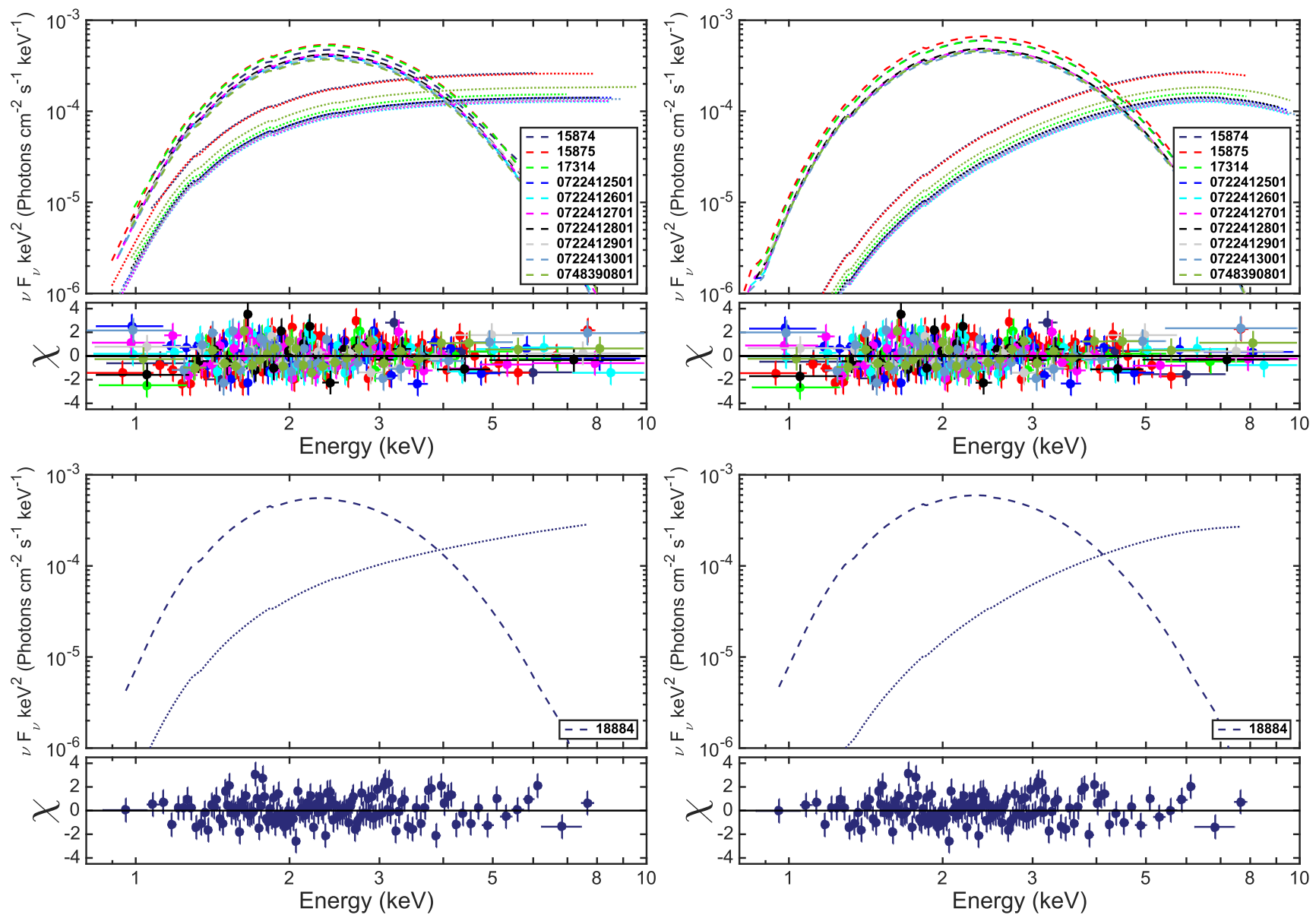

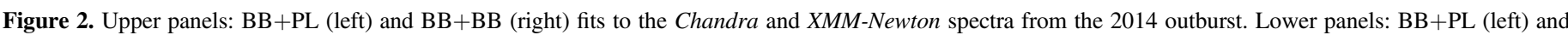

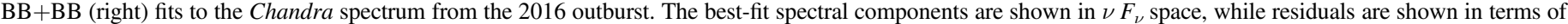
$\chi$. See the text and Table 3 for details.

$\mathrm{BB}$ temperature $k T=0.51 \pm 0.04$, a $\mathrm{BB}$ emitting area radius $R=1.4_{-0.3}^{+0.5} \mathrm{~km}$, and a PL photon index $\Gamma=0.9 \pm 0.1$. This spectral fit results in $0.5-10 \mathrm{keV}$ and $10-50 \mathrm{keV}$ absorptioncorrected fluxes of $(2.6 \pm 0.4) \times 10^{-12} \mathrm{erg} \mathrm{s}^{-1} \mathrm{~cm}^{-2}$ and $(2.2 \pm 0.2) \times 10^{-12} \mathrm{erg} \mathrm{s}^{-1} \mathrm{~cm}^{-2}$, respectively. Table 4 summarizes the best-fit model parameters, while Figure 5 shows the data and best-fit model components in $\nu F \nu$ space (upper panel) and the residuals in terms of $\sigma$ (lower panel).

Since the Chandra and XMM-Newton 2014 spectra were best fit with a two-component model below $10 \mathrm{keV}$, we added a third component to the Swift $+N u S T A R$ data, a BB or a PL. Such a three-component modelis required for many bright magnetars to fit the broadband $0.5-79 \mathrm{keV}$ spectra (e.g., Hascoët et al. 2014). For SGR J1935+2154, the addition of either component does not significantly improve the quality of the fit, both resulting in a C-stat of 441 for 450 d.o.f. To understand whether our Swift $+N u S T A R$ data are of high enough $\mathrm{S} / \mathrm{N}$ to exclude the possibility of a three-component model, we simulated 10,000 Swift-XRT and NuSTAR spectra with their true exposure times, based on the $20140.5-10 \mathrm{keV}$ spectrum and including a hard PL component as measured above. We find that we cannot retrieve all three components at the $3 \sigma$ level; most of these simulated spectra are best fit with a two-component model, namely an absorbed PL + BB.

To study the spectral evolution of the source during its 2015 outburst, we fit the Swift/XRT spectra of observations taken after 2015 February 22 (Table 1) with an absorbed BB+PL and a 2BB model. We fixed the absorption column density, temperatures, and the photon index to the values derived with the 2014 XMM-Newton +Chandra fits, but allowed for them to vary within their $3 \sigma$ uncertainties in the case of a statistically bad fit. We show in Figure 3 the flux evolution of the BB+PL model and in Figure 4 the area evolution of the 2BB model. These results are discussed in Section 4.

\subsubsection{The 2016 Outburst}

We started our spectral analysis of the 2016 outburst with the Chandra observation taken on July 07. Similar to the high-S/N spectra from the 2014 and 2015 outbursts, an absorbed BB or PL spectral model fails to describe the data adequately. Hence, we fit an absorbed BB+PL and a $2 \mathrm{BB}$ model to the data. Both models result in equally good fits with a C-stat of 289 for 302 d.o.f. The best-fit model parameters are shown in Table 3, while the models in $\nu F_{\nu}$ space and deviations of the data from the model in terms of $\sigma$ are shown in Figure 5. These spectral parameters are within $1 \sigma$ uncertainty from the parameters derived during the 2014 and 2015 outbursts.

SGR J1935+2154 was observed regularly after the May outburst of 2016 with Swift. These observations also covered its 2016 June outburst. We analyzed all XRT observations taken during this period and fit all spectra with an absorbed BB $+\mathrm{PL}$ and a $2 \mathrm{BB}$ model. We froze the absorption hydrogen column density $N_{\mathrm{H}}, \Gamma$, and $k T$ to the best-fit values as derived during 


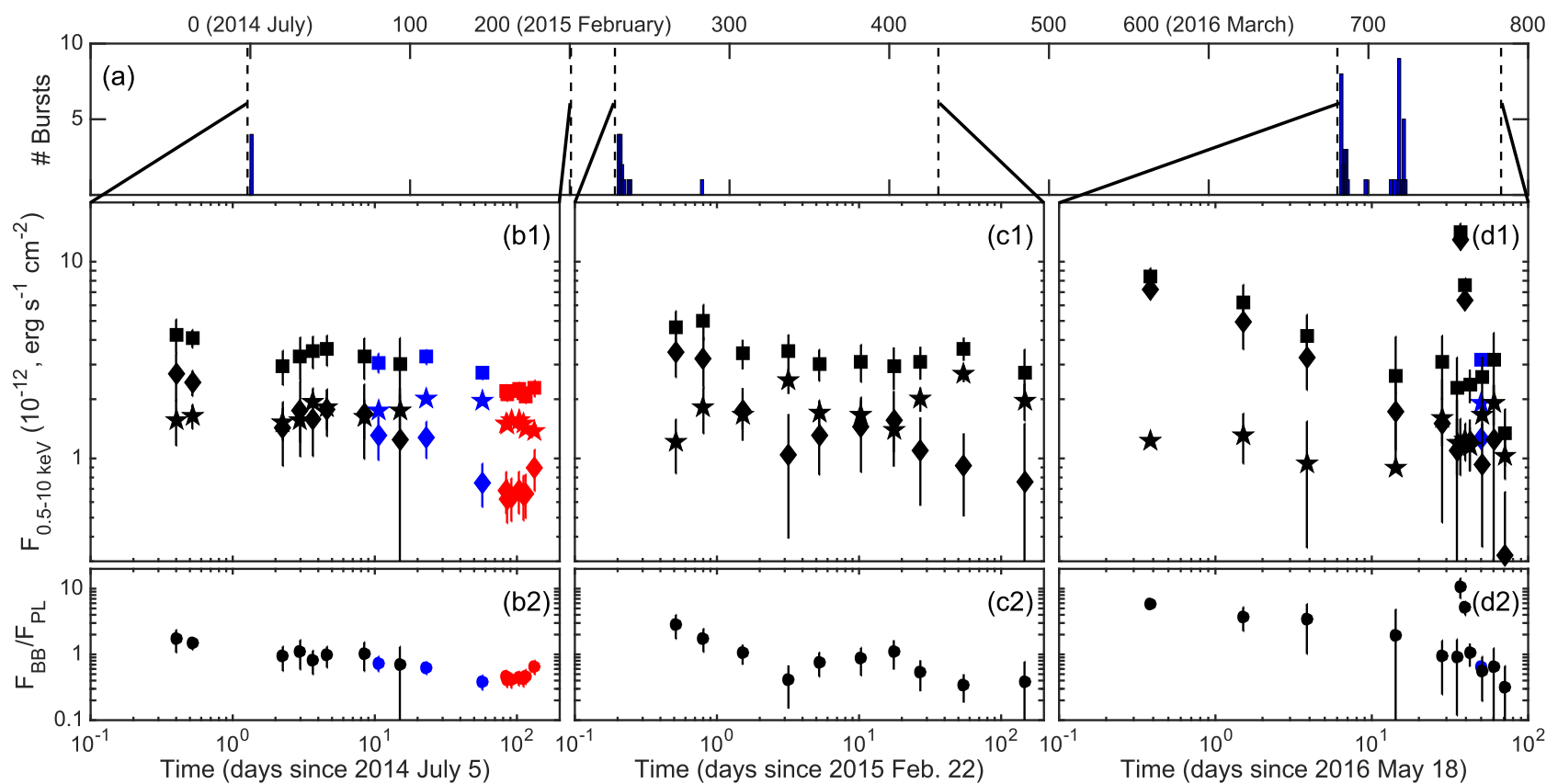

Figure 3. SGR J1935+2154 BB+PL spectral evolution during the 2014, 2015, and 2016 May and June outbursts. Panel (a) shows the number of bursts detected by the Inter Planetary Network (IPN) since the source discovery and up to 2016 August. Panels (b1), (c1), and (d1) represent the evolution of the BB (stars), PL (diamonds), and total fluxes (squares) from outburst onset and up to 200 days. Panels (b2), (c2), and (d2) represent the evolution of the $F_{\mathrm{PL}} / F_{\mathrm{BB}}$ ratio. Colors represent fluxes derived from different instruments (black:Swift, blue:Chandra, red:XMM-Newton). See the text for details.

the 2014 outburst. The evolution of the flux for the BB+PL model and the emitting area radius of the 2BB model are shown in Figures 3 and 4, respectively.

\subsection{Outburst Comparison and Evolution}

We first concentrate on the 2014 outburst, which has the best observational coverage compared to the rest. The outburst decay is best fit with an exponential function $F(t)=$ $K e^{-t / \tau}+F_{\mathrm{q}}$, where $K$ is a normalization factor, while $F_{\mathrm{q}}=2.1 \times 10^{-12} \mathrm{erg} \mathrm{s}^{-1} \mathrm{~cm}^{-2}$ is the assumed quiescent flux level as derived with the XMM-Newton observations (Figure 6). This fit results in a characteristic decay timescale $\tau_{14}=29 \pm 4$ days (Table 5). Integrating over 200 days, we find a total energy in the outburst, corrected for the quiescent flux level, of $E_{14}=(4.1 \pm 0.7) \times 10^{40} \mathrm{erg}$. We find a flux at outburst onset $F_{\text {on-14 }}=(4.3 \pm 0.7) \times 10^{-12} \mathrm{erg} \mathrm{s}^{-1} \mathrm{~cm}^{-2}$ and a ratio to the quiescent flux level $R_{14} \approx 2.0$. Following the same recipe for the 2015 outburst, we find a characteristic decay timescale $\tau_{15}=43_{-8}^{+12}$ days and a total energy in the outburst, corrected for the quiescent flux level, $E_{15}=(6.1 \pm 1.1) \times 10^{40} \mathrm{erg}$. The flux at outburst onset is $F_{\text {on-15 }}=(4.7 \pm 0.08) \times$ $10^{-12} \mathrm{erg} \mathrm{s}^{-1} \mathrm{~cm}^{-2}$, and its ratio to the quiescent flux level $R_{15}=2.2$.

A similar analysis for the 2016 May and June outbursts was difficult to perform because of the lack of observations $\sim 30$ days beyond the start of each outburst (Figure 6) and the poor constraints on the fluxes (due to the short XRT exposures) derived a few days after the outburst onset. These fluxes are consistent with $F_{\mathrm{q}}$ and the slightly brighter flux level seen in the 2014 and 2015 outbursts between a few days after outburst onset and the quiescence reached $\sim 70$ days later. Hence, we cannot derive the long-term decay shape of the light curve during the last two outbursts from SGR J1935+2154.

However, an exponential-decay fit to the 2016 outbursts results in short-term characteristic timescales $\tau_{\text {May-16 }} \approx \tau_{\text {June-16 }} \approx 4$ days, indicating a quick initial decay that might have been followed by a longer one similar to what was observed in 2014 and 2015. To enable a comparison between all outbursts, we derive the total energy emitted within 10 days of each outburst. These are reported in Table 5. The 2016 outburst onset to quiescence flux ratios are $R_{\text {May16 }}=4.0$ and $R_{\text {June16 }}=6.7$. Table 5 also includes the total energy in the bursts during the first day of each of the outbursts (L. Lin et al. 2017, in preparation).

The decay timescales and total energies in the outbursts are derived assuming a quiescent flux level consistent with the late $X M M-N e w t o n$ observations. If, however, the true quiescent flux level of SGR J1935+2154 is lower (e.g., Israel et al. 2016b), this would increase the decay timescale and the total energy corrected for the energy released in the persistent emission. In such case, the light curve decay shape would probably be more complicated than a simple exponential function. Continued monitoring of the source is important to alleviate these systematic uncertainties on the source outburst properties.

Finally, we note that the last observation during the 2016 May outburst was taken 1.5 days prior to the start of the June outburst (last green dot and first red square in Figure 6). The total fluxes from the two observations differ at the $\gtrsim 5 \sigma$ level. These results are discussed in Section 4.2.

\section{Discussion}

\subsection{Broadband X-Ray Properties}

Using high-S/N observations, we have established that the SGR J1935+2154 soft X-ray spectrum, with photon energies $<10 \mathrm{keV}$, is well described with the phenomenological BB + PL or $2 \mathrm{BB}$ model. NUSTAR observations, on the other hand, were crucial in providing the first look at this magnetar at energies $>10 \mathrm{keV}$, revealing a hard X-ray tail extending up to $50 \mathrm{keV}$. We note that this NUSTAR observation was taken five days after the 2015 outburst. The Swift fit revealed a 


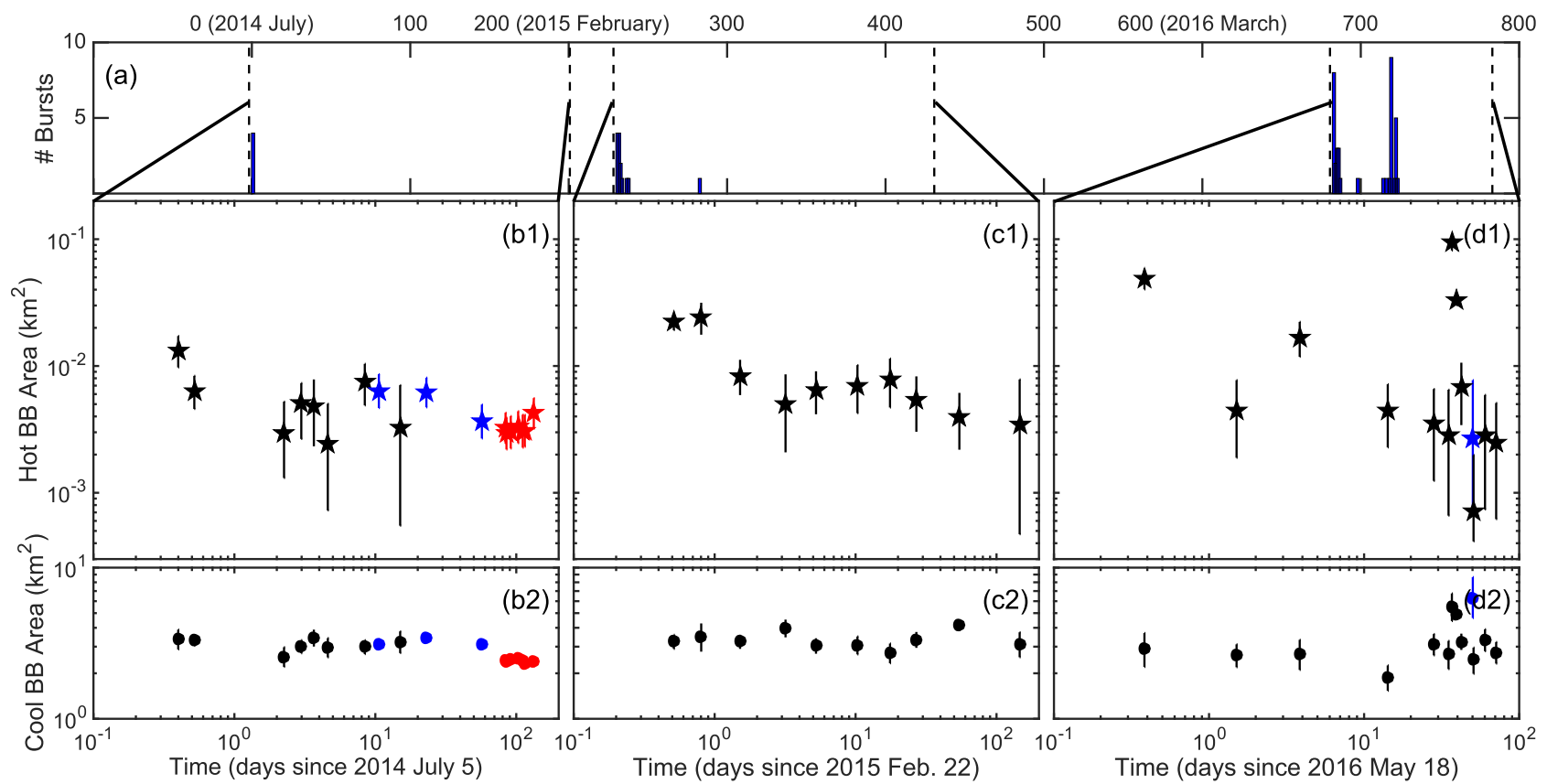

Figure 4. SGR J1935+2154 BB+BB spectral evolution during the 2014, 2015, and 2016 May and June outbursts. Panel (a) shows the number of bursts detected by the Inter Planetary Network (IPN) since the discovery and up to 2016 August. Panels (b1), (c1), and (d1) represent the evolution of the hot BB area from outburst onset and up to 200 days. Panels (b2), (c2), and (d2) represent the evolution of the cool BB area. Colors represent values derived from different instruments (black:Swift, blue:Chandra, red:XMM-Newton). See the text for more details.

Table 4

Best-fit Spectral Parameters to the 2015 Simultaneous Swift-XTR and NuSTAR Spectra

\begin{tabular}{|c|c|}
\hline \multicolumn{2}{|c|}{$\mathrm{BB}+\mathrm{PL}$} \\
\hline$N_{\mathrm{H}}\left(10^{22} \mathrm{~cm}^{-2}\right)$ & $2_{-0.7}^{+0.8}$ \\
\hline$k T(\mathrm{keV})$ & $0.51 \pm 0.04$ \\
\hline$R_{\text {cool }}{ }^{\mathrm{a}}(\mathrm{km})$ & $1.4_{-0.3}^{+0.5}$ \\
\hline$F_{\mathrm{BB}}\left(10^{-12} \mathrm{erg} \mathrm{s}^{-1} \mathrm{~cm}^{-2}\right)$ & $1.6_{-0.4}^{+0.5}$ \\
\hline$\Gamma$ & $0.9 \pm 0.1$ \\
\hline$F_{\mathrm{PL}}\left(10^{-12} \mathrm{erg} \mathrm{s}^{-1} \mathrm{~cm}^{-2}\right)$ & $2.3_{-0.3}^{+0.5}$ \\
\hline$F_{0.5-10 \mathrm{keV}}\left(10^{-12} \mathrm{erg} \mathrm{s}^{-1} \mathrm{~cm}^{-2}\right)$ & $2.6 \pm 0.4$ \\
\hline$F_{10-50 \mathrm{keV}}\left(10^{-12} \mathrm{erg} \mathrm{s}^{-1} \mathrm{~cm}^{-2}\right)$ & $2.2_{-0.1}^{+0.2}$ \\
\hline 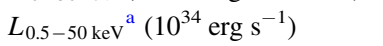 & $4.1 \pm 0.3$ \\
\hline
\end{tabular}

Note.

${ }^{\mathrm{a}}$ Assuming a distance of $9 \mathrm{kpc}$.

$0.5-10 \mathrm{keV}$ flux $\sim 40 \%$ larger than the quiescent flux, which we assume to be at the 2014 XMM-Newton level of $2.2 \times 10^{-12}$ $\mathrm{erg} \mathrm{s}^{-1} \mathrm{~cm}^{-2}$. The spectra below $10 \mathrm{keV}$ did not show significant spectral variability during any of the outbursts (Section 4.2), except for the relative brightness. Accordingly, one can conjecture that SGR J1935+2154 has a similar highenergy tail during quiescence, though proof of such requires further dedicated monitoring of the source with NUSTAR or INTEGRAL.

The presence of hard X-ray tails, such as exhibited by SGR J1935+2154, is clearly seen in about one-third of all known magnetars (e.g., Kuiper et al. 2006; Esposito et al. 2007; den Hartog et al. 2008b; Enoto et al. 2010), but may indeed be universal to them. Spectral details differ across the population. For instance, the hard X-ray tail photon index we measure, $\Gamma_{\mathrm{H}} \approx 0.9$, is quite similar to some measured for AXPs (e.g., den Hartog et al. 2008a; An et al. 2013; Vogel et al. 2014;

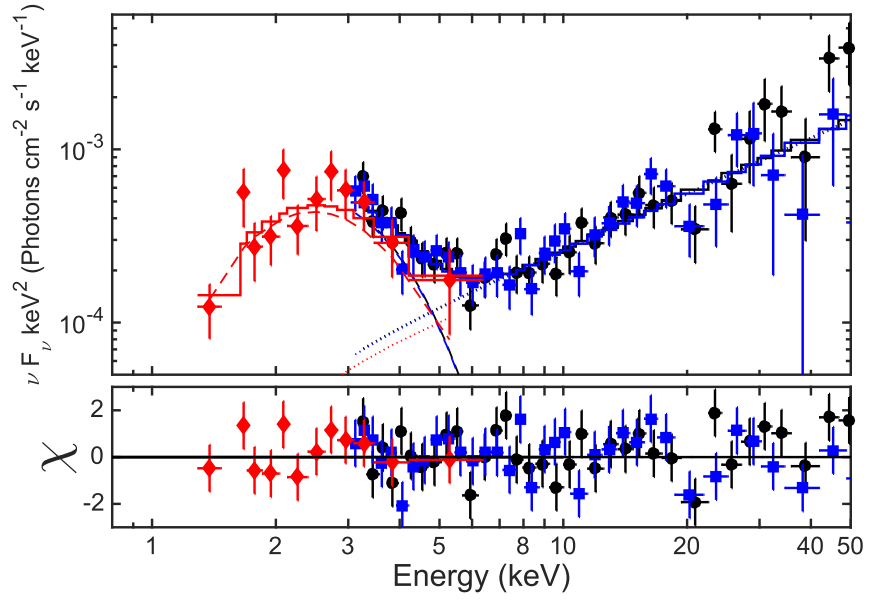

Figure 5. Upper panel: simultaneous broadband NUSTAR and Swift-XRT spectra of SGR J1935+2154 taken on 2015 February 27, five days after the 2015 outburst onset. Dots, squares, and diamonds are the NuSTAR FPMA, FPMB, and Swift-XRT spectra, respectively. The solid lines represent the absorbed $\mathrm{BB}+\mathrm{PL}$ best-fit model in $\nu F \nu$ space, while the dashed and dotted lines represent the $\mathrm{BB}$ and $\mathrm{PL}$ components, respectively. Lower panel: residuals of the best fit are shown in terms of standard deviation.

Tendulkar et al. 2015), but somewhat harder than for other sources (e.g., Esposito et al. 2007; Yang et al. 2016). Moreover, the flux in the hard PL tail is 1.5 times larger than the flux in the soft components. This flux ratio varies by about two orders of magnitude among the magnetar population (Enoto et al. 2010).

Kaspi \& Boydstun (2010, see also Marsden \& White 2001; Enoto et al. 2010) searched for correlations between the observed X-ray parameters and the intrinsic parameters for magnetars. They found an anticorrelation between the index differential $\Gamma_{\mathrm{S}}-\Gamma_{\mathrm{H}}$ and the strength of the magnetic field $B$. For 


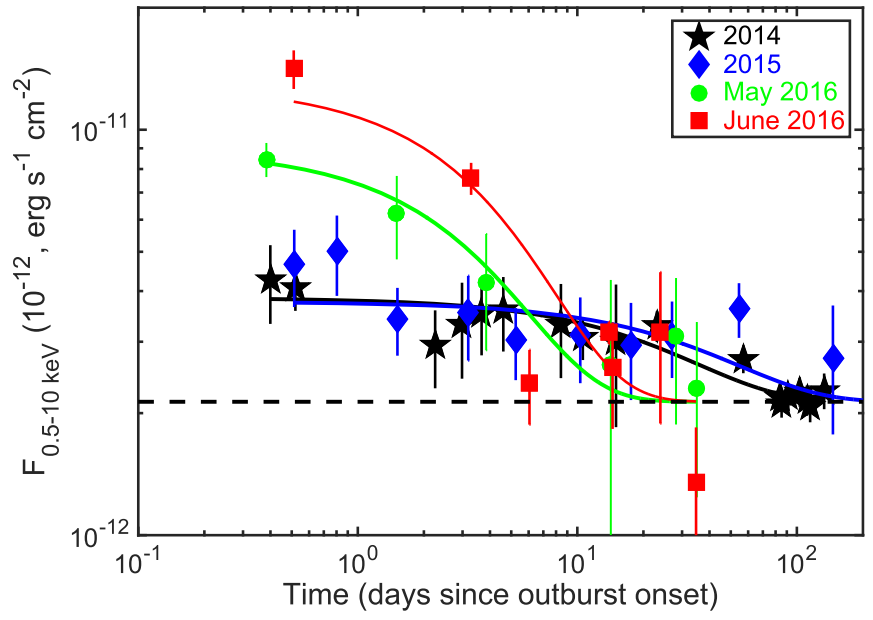

Figure 6. Total $0.5-10 \mathrm{keV}$ flux evolution with time for all four outbursts detected from SGR J1935+2154. The flux level reached the highest at outburst onset during the latest outburst of 2016 June, during which the largest number of bursts have been detected from the source. Solid lines represent an exponential-decay fit. See the text for details.

SGR J1935+2154, with its spin-down field strength of $B=2.2 \times 10^{14} \mathrm{G}$ (Israel et al. 2016b), the determination here of $\Gamma_{\mathrm{S}}-\Gamma_{\mathrm{H}} \approx 1.0-2.0$ nicely fits the Kaspi \& Boydstun (2010) correlation. Moreover, Enoto et al. (2010) noted a strong correlation between the hardness ratio, defined as $F_{\mathrm{H}} / F_{\mathrm{S}}$ for the hard and soft energy bands, respectively, and the characteristic age $\tau$. Following the same definition for the energy bands as in Enoto et al. (2010), we find $F_{\mathrm{H}} / F_{\mathrm{S}} \approx 1.4$, which falls very close to this correlation line given the SGR J1935+2154 spindown age $\tau=3.6 \mathrm{Kyr}$ (Israel et al. 2016b). Since the electric field for a neutron star $E$ along its last open field line is nominally inversely proportional to the characteristic spindown age $E=\Omega R B \propto \tau^{-1 / 2}$, Enoto et al. (2010) argued that a younger magnetar will be able to sustain a larger current, accelerating more particles into the magnetosphere and causing a stronger hard X-ray emission in the tail. This scenario is predicated on the conventional picture of powerful, young rotation-powered pulsars like the Crab.

The most discussed model for generating a hard X-ray tail in magnetar spectra is resonant Compton up-scattering of soft thermal photons by highly relativistic electrons with Lorentz factors $\sim 10-10^{4}$ in the stellar magnetosphere (e.g., Baring \& Harding 2007; Fernández \& Thompson 2007; Beloborodov 2013). The emission locale is believed to be at distances $\sim 10-100 R_{\mathrm{NS}}$ where $R_{\mathrm{NS}}=10 \mathrm{~km}$ is the NS radius. There the intense soft X-ray photon field seeds the inverse Compton mechanism, and the collisions are prolific because of scattering resonances at the cyclotron frequency and its harmonics in the rest frame of an electron. Magnetar conditions guarantee that electrons accelerated by voltages in the inner magnetosphere will cool rapidly down to Lorentz factors $\gamma \sim 10-10^{2}$ (Baring et al. 2011) due to the resonant scatterings. Along each field line, the up-scattered spectra are extremely flat, with indices $\Gamma_{h} \sim-0.5-0.0$ (Baring \& Harding 2007; see also Wadiasingh et al. 2017), though the convolution of contributions from extended regions is necessarily steeper and more commensurate with the observed hard tail spectra (Beloborodov 2013). While the inverse Compton emission can also extend out to gamma-ray energies, the prolific action of attenuation mechanisms such as magnetic pair creation $\gamma \rightarrow e^{+} e^{-}$and photon splitting $\gamma \rightarrow \gamma \gamma$ (Baring \& Harding 2001) limits emergent signals to energies below a few megaelectronvolts in magnetars (Story \& Baring 2014), and probably even below $500 \mathrm{keV}$.

Beloborodov (2013, see also Chen \& Beloborodov 2017) developed a coronal outflow model based on the above picture, using the twisted magnetosphere scenario (Thompson et al. 2002; Beloborodov 2009). Twists in closed magnetic field loops (dubbed $J$-bundles) extending high into the magnetosphere can accelerate particles to high Lorentz factors, which will decelerate and lose energy via resonant Compton up-scattering. If pairs are created in profusion, they then annihilate at the top of a field loop. Another one of the $J$-bundle model predictions is a hot spot on the surface formed when return currents hit the surface at the footprint of the twisted magnetic field lines. The physics in this model is mostly governed by the field line twist amplitude $\psi$ (Thompson et al. 2002), the voltage $\Phi_{j}$ in the bundle, and its half-opening angle to the magnetic axis $\theta_{\mathrm{j}}$ (Beloborodov 2013; Hascoët et al. 2014).

The temperatures expected for the hot spots are of the order of $\sim 1 \mathrm{keV}$, while areas depend on the geometry of the bundle and the angle $\theta_{j}$. For a dipole geometry, $A_{\mathrm{j}} \sim$ (1/4) $\theta_{\mathrm{j}}^{2} A_{\mathrm{ns}} \approx 0.02\left(\theta_{\mathrm{j}} / 0.3\right)^{2} A_{\mathrm{ns}}$, where $A_{\mathrm{ns}}=4 \pi R_{\odot}^{2}$ is the NS surface area (Hascoët et al. 2014). Assuming that the hot BB in our model discussed in the last paragraph of Section 3.3.1 represents the footprints of the $J$-bundle, for which we find a temperature $k T=0.8 \mathrm{keV}$, we estimate its surface area $A \approx$ $0.6 \mathrm{~km}^{2}$. Assuming that $A \approx A_{\mathrm{j}}$, we estimate $\theta_{\mathrm{j}} \approx 0.05$.

The above calculation assumes that the $J$-bundle is axisymmetric extending all around the NS. The hot spot, hence, is a ring around the polar cap rim. The smaller area that we derive may suggest that the $J$-bundle is not axisymmetric and extends only around part of the NS, implying that the twist could have been imparted onto local magnetic field lines.

The total power dissipated by the $J$-bundle in the twisted magnetosphere model can be expressed as $L_{\mathrm{j}} \approx 2 \times$ $10^{35} \psi \Phi_{10} \mu_{32} R_{10} \theta_{\mathrm{j}, 0.3}^{4} \mathrm{erg} \mathrm{s}^{-1}$ (Equation (3), Hascoët et al. 2014), where $\Phi_{10}$ is the voltage in units of $10^{10} \mathrm{~V}, \mu_{32}$ is the magnetic moment in units of $10^{32} \mathrm{G} \mathrm{cm}^{3}, R_{10}$ is the NS radius in units of $10 \mathrm{~km}$, and $\theta_{\mathrm{j}, 0.3}=\theta_{\mathrm{j}} / 0.3$. Given the magnetic moment of SGR J1935+2154, for choices of $\phi_{10}=1, \psi=1$, $R_{10}=1$, and $\theta_{\mathrm{j}, 0.3} \approx 0.2$, we estimate $L_{\mathrm{j}}=7 \times 10^{32} \mathrm{erg} \mathrm{s}^{-1}$. This luminosity is a factor of $\sim 17$ smaller than the hard tail PL luminosity, $L_{\mathrm{PL}}=1.1 \times 10^{34} \mathrm{erg} \mathrm{s}^{-1}$, we derive with the NUSTAR data, after normalizing it to the 2014 XMM-Newton flux level. ${ }^{19}$ This might imply a larger voltage across the twisted field lines than the choice of $\phi_{10}=1$, which corresponds to only $\sim 3 \times 10^{-6}$ times the open field line pole-to-equator voltage $2 \pi R_{\mathrm{NS}}^{2} B_{p} /(P c) \approx 2.8 \times 10^{16} \mathrm{~V}$ for SGR J1935+2154. Another possibility is that the hard PL tail could be much fainter during quiescence, which might indicate a different decay trend for the high-energy tail compared to the 0.5-10 keV spectrum. A deep XMM-Newton $+N u S T A R$ observation of SGR J1935+2154 during quiescence would help reveal the exact shape and power of the hard PL tail, inform on how activation relates to heat transfer to and from the stellar surface layers, and help refine the twisted magnetosphere model.

\footnotetext{
19 The NUSTAR observation was taken five days after the outburst when the simultaneous XRT observation showed an increase in the PL flux a factor of 2 above the quiescent XMM-Newton level of 2014. We normalized the hard PL luminosity from Table 4 by the same factor. See also footnote 17.
} 
Table 5

Outburst Properties

\begin{tabular}{lccccr}
\hline \hline Outburst & $\begin{array}{c}\tau \\
(\text { days })\end{array}$ & $\begin{array}{c}K \\
\left(10^{-12}\right)\end{array}$ & $\begin{array}{c}E_{10}^{\mathrm{a}} \\
\left(10^{40} \mathrm{erg}\right)\end{array}$ & $\begin{array}{c}E_{200}{ }^{\mathrm{b}} \\
\left(10^{40} \mathrm{erg}\right)\end{array}$ & $\begin{array}{c}F_{\text {peak }} \\
\left(10^{-12} \mathrm{erg} \mathrm{s}^{-1} \mathrm{~cm}^{-2}\right)\end{array}$ \\
\hline 2014 & $29 \pm 4$ & $1.7 \pm 0.2$ & $1.2 \pm 0.3$ & $4.1 \pm 0.7$ & $4.3 \pm 0.7$ \\
2015 & $43_{-8}^{+12}$ & $1.6_{-0.3}^{+0.4}$ & $1.2 \pm 0.2$ & $6.1 \pm 1.1$ & $4.7 \pm 0.8$ \\
2016 May $^{\mathrm{d}}$ & $3.7 \pm 1.0$ & $6.8_{-0.5}^{+0.7}$ & $2.0 \pm 0.3$ & $\mathrm{NA}$ & $8.5 \pm 0.6$ \\
2016 June $^{\mathrm{d}}$ & $4.3 \pm 1.0$ & $10.8_{-2.5}^{+3.2}$ & $3.6 \pm 0.4$ & $\mathrm{NA}$ & $14 \pm 1$ \\
\hline
\end{tabular}

Notes. All energies are derived assuming a distance of $9 \mathrm{kpc}$.

${ }^{a}$ Integrated total energy within 10 days from outburst onset.

${ }^{\mathrm{b}}$ Integrated total energy within 200 days from outburst onset.

${ }^{\mathrm{c}}$ Total energy in the bursts for the day of the outburst onset, i.e., 2014 July 05, 2015 February 22, 2016 May 18, 2016 June 23 (L. Lin et al. 2017 , in preparation).

${ }^{\mathrm{d}}$ Long-term outburst behavior during 2016 cannot be explored due to a lack of high-S/N observations beyond a few days of outburst onset. See the text for details.

\subsection{Outbursts}

Since its discovery in 2014 June, SGR J1935+2154 has shown four major bursting episodes, which culminated with the strongest one to date in 2016 June. Similar to most other magnetars, SGR J1935+2154 bursting activity was accompanied by a persistent emission outburst, showing an increase in the flux level at, or shortly after, the onset of the bursting activity that decayed quasi-exponentially back to quiescence (e.g., Woods et al. 2004; Gögüuş et al. 2010; Rea \& Esposito 2011; Kargaltsev et al. 2012; Scholz et al. 2012; Coti Zelati et al. 2015; Younes et al. 2015b).

The rise time of magnetar outbursts is a challenging observational property to identify and quantify due to the randomness of the process. Magnetars are usually observed by pointed XRTs after they have entered a bursting episode. Hence, it is unclear whether magnetars show any persistent flux enhancement prior to the bursting activity, or whether the two happen (quasi-)simultaneously. CXOU J164710.2-455216 was observed with XMM-Newton five days prior to bursting activity (Israel et al. 2007), as part of a monitoring program. The flux of this observation was consistent with quiescence, while the following observation, which took place less than a day after the bursts, showed an increase by a factor of $\sim 300$. A serendipitous Chandra observation of SGR 1627-41, $20 \mathrm{hr}$ before its 2008 bursting episode, set an upper limit that, although larger than its true quiescent level, was a factor of a few lower than the flux measured shortly after outburst onset. This indicates that most of the energy in the outburst was indeed emitted in concordance with the bursting activity (Esposito et al. 2008). SGR J1935+2154 was observed $\sim 1.5$ days prior to its strongest bursting activity in 2016 June while being monitored for its 2016 May activation. The latter observation showed a flux level close to quiescence and was $5 \sigma$ away from the flux measured at the start of the 2016 June outburst (Section 3.4). Hence, the behavior of SGR J1935 +2154 , along with the other mentioned magnetars, implies that any instability invoked to explain the outbursts in magnetars has to develop on very short timescales $(\lesssim 2$ days, e.g., Li et al. 2016).

The $0.5-10 \mathrm{keV}$ persistent flux level of SGR J1935+2154 at or shortly after the onset of the bursting activity varied in concordance with the bursting level from the source (see also, e.g., 1E 1547.0-5408, Ng et al. 2011). The source flux reached its highest level at the start of the 2016 June outburst, a factor of 7 of the quiescent level (Figure 6). At the same time, the flux of the PL or the hot BB components (Figures 3 and 4) increased by a factor of $\sim 25$ compared to quiescence. The cold $\mathrm{BB}$, on the other hand, with a temperature of $k T=0.48 \mathrm{keV}$ and radius $R=1.8 \mathrm{~km}$, remained more or less constant throughout all four outbursts. Such a cold BB could be the result of internal heating of a large fraction of the magnetar surface (Thompson \& Duncan 1996; Beloborodov \& Li 2016).

The 2014 and 2015 flux decays followed a simple exponential trend with timescales of $\sim 30-40$ days. The brighter 2016 outbursts, however, exhibited a quick decay trend on timescales of about four days. Such a fast initial drop in flux is seen at the outburst onset of a number of magnetars (e.g., SGR J1627-41, An et al. 2012; Swift J1834.9-0846, Kargaltsev et al. 2012; Swift J1822.3-1606, Scholz et al. 2012).

A similar amount of energy was emitted in the 2014 and 2015 outbursts (within $2 \sigma$ ), $E \sim 5 \times 10^{40} \mathrm{erg} \mathrm{s}^{-1}$. We were only able to quantify the total energy emitted during the first 10 days of the 2016 May and June outbursts, $E=2 \times 10^{40} \mathrm{erg} \mathrm{s}^{-1}$ and $E=3.6 \times 10^{40} \mathrm{erg} \mathrm{s}^{-1}$, respectively. The energetics in these outbursts are at the lower end compared to the bulk of magnetar outbursts (Rea \& Esposito 2011). We note that the energy in the bursts for the four outbursts varied by more than two orders of magnitude (Table 5, L. Lin et al. 2017, in preparation); a much larger increase than the energy emitted in the outbursts. For instance, the 2014 and 2015 ratios of total energy in the outbursts to total energy in bursts decreased from 50 to 8 .

Two models have been discussed in the context of magnetar outbursts. The first invoked an instability (external or internal) that rapidly (within a few days) deposits energy, of the order of $10^{40}-10^{42} \mathrm{erg} \mathrm{s}^{-1}$, at the crust level of the NS (e.g., Lyubarsky et al. 2002; Brown \& Cumming 2009; Pons \& Rea 2012). The depth at which the heat is deposited governs the outburst decay timescale, which can range from weeks to months, as the crust cools back to its preoutburst level. This timescale may also reflect the magnetic colatitude of the energy dissipation locale, as heat conductivity across strong fields is suppressed, so that vertical transport of energy is easier in polar activation zones. This picture fits the observed properties of the 2014 and 2015 outbursts of SGR J1935+2154. It is, however, difficult to reconcile the initial quick decay of a few days observed in the 2016 outbursts, and in a number of other magnetars, with this model.

In the second theoretical picture, magnetar outbursts are believed to be triggered when stresses on the crust build up to a critical level due to Hall wave propagation caused by magnetic 


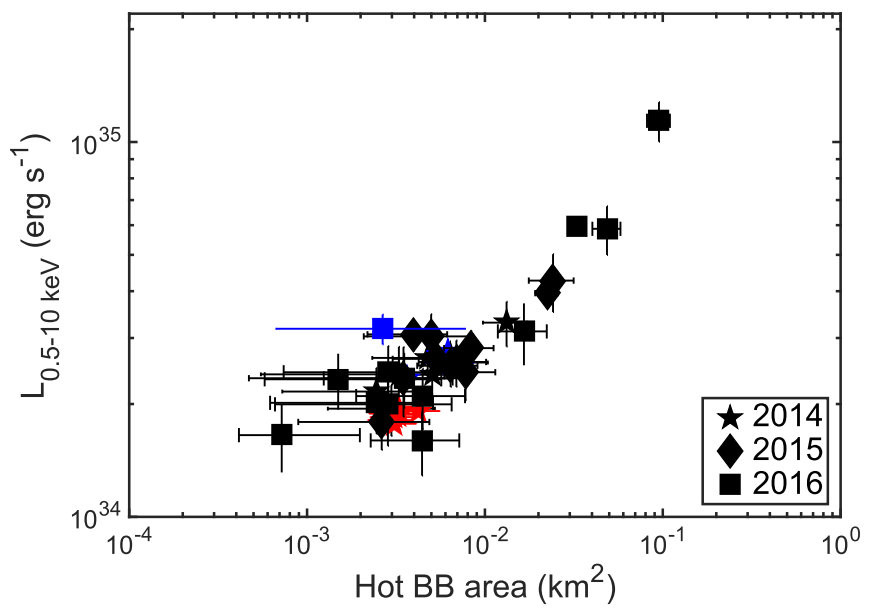

Figure 7. Total $0.5-10 \mathrm{keV}$ flux versus hot $\mathrm{BB}$ area from all outbursts of SGR J1935+2154. See also Figure 4.

field evolution inside the NS (e.g., Thompson \& Duncan 1996; Pons \& Rea 2012; Li et al. 2016). These stresses twist a bundle of external magnetic field lines anchored to the surface, accelerating particles off the surface of the star, while returning currents deposit heat at the footprints of these lines (hot spot; Thompson et al. 2002; Beloborodov 2009). This instability develops on a timescale of days to weeks( $\mathrm{Li}$ et al. 2016), with decay timescales ranging from weeks to years and primarily depending on the strength of the twist imparted onto the B-field bundle. These properties match the outburst properties that we observe for SGR J1935+2154. Another prediction of this model is a shrinking hot spot at the surface, which we do observe when we fit the $0.5-10 \mathrm{keV}$ spectra with the $2 \mathrm{BB}$ model $^{20}$ (Figure 7). However, similar to the crust heating model, it is not trivial to explain the initial quick decay observed in the 2016 outbursts with the twisted magnetosphere model.

\subsection{Radio Comparison to Other Magnetars}

The upper limits on the radio counterpart that we have obtained are the deepest radio limits for SGR J1935+2154 thus far (i.e., Surnis et al. 2016). In fact, our Arecibo observations represent the deepest radio observations that were carried out quickly after the X-ray outburst of a magnetar (e.g., Crawford et al. 2007; Lazarus et al. 2012). Currently it is not clear what is the best epoch to search for magnetar radio emission. The sample of magnetars with radio detections is small, and although some were detected close in time to their X-ray activation (e.g., Anderson et al. 2012; Scholz et al. 2017), there does not seem to be a clear correlation between magnetar X-ray and radio activity, with the latter sometimes switching on and off during an outburst (Burgay et al. 2009). We note in this context that the SGR J1935+2154 spin-down luminosity of $1.7 \times 10^{34} \mathrm{erg} \mathrm{s}^{-1}$ and $\mathrm{X}$-ray luminosity in quiescence of $2.1 \times 10^{34} \mathrm{erg} \mathrm{s}^{-1}(0.5-10 \mathrm{keV})$ put SGR J1935+2154 in the area of magnetars that are not expected to display radio emission in the fundamental plane of Rea et al. (2012). The latter needs to be tested further with deep radio searches, like

\footnotetext{
${ }^{20}$ We do not attempt to quantitatively compare the flux-versus-area relation we observe here to the prediction of Beloborodov (2009), due to uncertainties in the parameter estimates of the 2BB model discussed in Section 3.3.1.
}

those presented in this paper, both when magnetars are X-ray active and when they are in their quiescent state.

\section{Conclusions}

In the following we summarize the main findings of our analyses of the broadband X-ray and radio data of the magnetar SGR J1935+2154 taken in the aftermath of its 2014, 2015, and 2016 outbursts:

1. Chandra data did not reveal any small-scale extended emission around SGR J1935+2154.

2. No pulsations are detected from SGR J1935+2154 in the days following the 2015 and 2016 outbursts. We derive an upper limit of $25 \%$ and $8 \%$ in the energy range 3-50 keV during 2015 and 1-8 keV during 2016 with NUSTAR and Chandra, respectively. These upper limits are consistent with the pulsed fractions derived in Israel et al. (2016b).

3. No persistent radio pulsations or sporadic single pulses are detected with Arecibo from SGR J1935+2154 following the 2015 and 2016 outbursts. We set the deepest limits on the radio emission from a magnetar, with a maximum flux density limit of $14 \mu \mathrm{Jy}$ for the $4.6 \mathrm{GHz}$ observations and $7 \mu \mathrm{Jy}$ for the $1.4 \mathrm{GHz}$ observations.

4. The soft X-ray spectrum $<10 \mathrm{keV}$ is well described with a $\mathrm{BB}+\mathrm{PL}$ or $2 \mathrm{BB}$ model during all three outbursts.

5. NuSTAR observations five days after the 2015 outburst onset revealed a hard X-ray tail, $\Gamma=0.9$, extending up to $79 \mathrm{keV}$, with a flux similar to the one detected $<10 \mathrm{keV}$.

6. Following the outbursts, the $0.5-10 \mathrm{keV}$ flux from SGR J1935+2154 increased in concordance to its bursting activity. At the onset of the 2016 June bursting episode, the strongest one to date, the $0.5-10 \mathrm{keV}$ reached maximum, increasing by a factor of $\sim 7$ above its quiescent level.

7. The $0.5-10 \mathrm{keV}$ flux increase during the outbursts is due to the PL or hot BB component, which increased by a maximum factor of 25 compared to quiescence. The cold BB component, $k T=0.47 \mathrm{keV}$, remained more or less constant.

8. The 2014 and 2015 outbursts decayed quasi-exponentially with timescales of $\sim 40$ days. The stronger 2016 May and June outbursts showed a quick short-term decay with timescales of about four days; their long-term decay trends were not possible to derive.

9. The last Swift/XRT observation of the 2016 May outburst, taken 1.5 days prior to the onset of the 2016 June outburst, showed a flux level close to quiescence and was dimmer at the $5 \sigma$ level compared to the flux measured at the start of the 2016 June outburst.

10. The total energy emitted by the bursts increased by two orders of magnitude between the 2014 and the 2016 June outbursts (Table 5, L. Lin et al. 2017, in preparation). This is a much larger increase compared to the energy emitted by the star through the increase of its X-ray persistent emission.

We thank NuSTAR PI Fiona Harrison and Belinda Wilkes for granting NuSTAR and Chandra DDT observations of SGR J1935+2154 during the 2015 and 2016 outbursts, respectively. We also thank the Swift team for performing the monitoring of the source during all of its outbursts. G.Y. 
and C.K. acknowledge support by NASA through grant NNH07ZDA001-GLAST. A.J. and J.W.T.H. acknowledge funding from the European Research Council under the European Union's Seventh Framework Programme (FP7/ 2007-2013) ERC grant agreement no. 337062 (DRAGNET). The Arecibo Observatory is operated by SRI International under a cooperative agreement with the National Science Foundation (AST-1100968) and in alliance with Ana G. Méndez-Universidad Metropolitana and the Universities Space Research Association. We would like to thank Arecibo observatory scheduler Hector Hernandez for the support during our observations. A.J. thanks Daniele Michilli for helping with the single-pulse search analysis. We thank the anonymous referee for their careful reading and insightful comments that improved the quality of the manuscript.

Software: CIAO (http://cxc.harvard.edu/ciao/), SAS (v14.0.0, https://www.cosmos.esa.int/web/xmmnewton/sas), xrtpipeline (v13.2), nustardas (v1.5.1, https://heasarc.gsfc. nasa.gov/docs/nustar/analysis/), HEASOFT (v6.20, https:// heasarc.nasa.gov/lheasoft/), ChaRT (http://cxc.harvard.edu/ ciao/PSFs/chart2/) MARX (http://space.mit.edu/CXC/ MARX/), PRESTO (Ransom 2001; Ransom et al. 2002, 2003), XSPEC (v12.9.0k; Arnaud 1996).

\section{ORCID iDs}

George Younes (10) https://orcid.org/0000-0002-7991-028X Chryssa Kouveliotou (iD https://orcid.org/0000-00031443-593X

Amruta Jaodand (ib https://orcid.org/0000-0002-3850-6651 Alexander J. van der Horst (ib https://orcid.org/0000-00019149-6707

Alice K. Harding (D) https://orcid.org/0000-0001-6119-859X

Jason W. T. Hessels (i) https://orcid.org/0000-0003-

2317-1446

Ramandeep Gill (10) https://orcid.org/0000-0003-0516-2968

Daniela Huppenkothen (10) https://orcid.org/0000-0002-

1169-7486

Jonathan Granot (ib https://orcid.org/0000-0001-8530-8941

Ersin Göğüş (DD https://orcid.org/0000-0002-5274-6790

\section{References}

An, H., Hascoët, R., Kaspi, V. M., et al. 2013, ApJ, 779, 163

An, H., Kaspi, V. M., Tomsick, J. A., et al. 2012, ApJ, 757, 68

Anderson, G. E., Gaensler, B. M., Slane, P. O., et al. 2012, ApJ, 751, 53

Antonopoulou, D., Weltevrede, P., Espinoza, C. M., et al. 2015, MNRAS, 447, 3924

Archibald, R. F., Kaspi, V. M., Tendulkar, S. P., \& Scholz, P. 2016, ApJL, $829, \mathrm{~L} 21$

Arnaud, K. A. 1996, in ASP Conf. Ser. 101, Astronomical Data Analysis Software and Systems V, ed. G. H. Jacoby \& J. Barnes (San Francisco, CA: ASP), 17

Baring, M. G., \& Harding, A. K. 1998, ApJL, 507, L55

Baring, M. G., \& Harding, A. K. 2001, ApJ, 547, 929

Baring, M. G., \& Harding, A. K. 2007, Ap\&SS, 308, 109

Baring, M. G., Wadiasingh, Z., \& Gonthier, P. L. 2011, ApJ, 733, 61

Beloborodov, A. M. 2009, ApJ, 703, 1044

Beloborodov, A. M. 2013, ApJ, 762, 13

Beloborodov, A. M., \& Li, X. 2016, ApJ, 833, 261

Bhattacharya, D. 1998, in The Many Faces of Neutron Stars, ed. R. Buccheri, J. van Paradijs, \& A. Alpar (Dordrecht: Kluwer), 103

Brown, E. F., \& Cumming, A. 2009, ApJ, 698, 1020

Buccheri, R., Bennett, K., Bignami, G. F., et al. 1983, A\&A, 128, 245

Burgay, M., Israel, G. L., Possenti, A., et al. 2009, ATel, 1913, 1

Burrows, D. N., Hill, J. E., Nousek, J. A., et al. 2005, SSRv, 120, 165

Camilo, F., Ransom, S. M., Halpern, J. P., et al. 2006, Natur, 442, 892
Camilo, F., Ransom, S. M., Halpern, J. P., \& Reynolds, J. 2007, ApJL, 666, L93

Chen, A. Y., \& Beloborodov, A. M. 2017, ApJ, 844, 133

Coti Zelati, F., Rea, N., Papitto, A., et al. 2015, MNRAS, 449, 2685

Crawford, F., Hessels, J. W. T., \& Kaspi, V. M. 2007, ApJ, 662, 1183 den Hartog, P. R., Kuiper, L., \& Hermsen, W. 2008a, A\&A, 489, 263

den Hartog, P. R., Kuiper, L., Hermsen, W., et al. 2008b, A\&A, 489, 245

Dewey, R. J., Taylor, J. H., Weisberg, J. M., \& Stokes, G. H. 1985, ApJL, 294, L25

Enoto, T., Nakazawa, K., Makishima, K., et al. 2010, ApJL, 722, L162 Esposito, P., Israel, G. L., Turolla, R., et al. 2011, MNRAS, 416, 205 Esposito, P., Israel, G. L., Zane, S., et al. 2008, MNRAS, 390, L34 Esposito, P., Mereghetti, S., Tiengo, A., et al. 2007, A\&A, 476, 321

Evans, P. A., Beardmore, A. P., Page, K. L., et al. 2007, A\&A, 469, 379

Evans, P. A., Beardmore, A. P., Page, K. L., et al. 2009, MNRAS, 397, 1177

Fernández, R., \& Thompson, C. 2007, ApJ, 660, 615

Gaensler, B. M. 2014, GCN, 16533, 1

Gavriil, F. P., Gonzalez, M. E., Gotthelf, E. V., et al. 2008, Sci, 319, 1802

Göğüş, E., Cusumano, G., Levan, A. J., et al. 2010, ApJ, 718, 331

Göğüş, E., Kouveliotou, C., Woods, P. M., Finger, M. H., \& van der Klis, M. 2002, ApJ, 577, 929

Göğüş, E., Lin, L., Kaneko, Y., et al. 2016, ApJL, 829, L25

Granot, J., Gill, R., Younes, G., et al. 2017, MNRAS, 464, 4895

Harrison, F. A., Craig, W. W., Christensen, F. E., et al. 2013, ApJ, 770, 103

Hascoët, R., Beloborodov, A. M., \& den Hartog, P. R. 2014, ApJL, 786, L1

Israel, G. L., Campana, S., Dall'Osso, S., et al. 2007, ApJ, 664, 448

Israel, G. L., Esposito, P., Rea, N., et al. 2016a, MNRAS, 457, 3448

Israel, G. L., Esposito, P., Rea, N., et al. 2016b, MNRAS, 457, 3448

Israel, G. L., Rea, N., Zelati, F. C., et al. 2014, ATel, 6370, 1

Israel, G. L., Romano, P., Mangano, V., et al. 2008, ApJ, 685, 1114

Kargaltsev, O., Kouveliotou, C., Pavlov, G. G., et al. 2012, ApJ, 748, 26

Kaspi, V. M., Archibald, R. F., Bhalerao, V., et al. 2014, ApJ, 786, 84

Kaspi, V. M., \& Boydstun, K. 2010, ApJL, 710, L115

Kozlova, A. V., Israel, G. L., Svinkin, D. S., et al. 2016, MNRAS, 460, 2008

Kuiper, L., Hermsen, W., den Hartog, P. R., \& Collmar, W. 2006, ApJ, 645, 556

Lazarus, P., Kaspi, V. M., Champion, D. J., Hessels, J. W. T., \& Dib, R. 2012, ApJ, 744, 97

Levin, L., Bailes, M., Bates, S., et al. 2010, ApJL, 721, L33

Li, X., Levin, Y., \& Beloborodov, A. M. 2016, ApJ, 833, 189

Liddle, A. R. 2007, MNRAS, 377, L74

Lin, L., Kouveliotou, C., Göğüs, E., et al. 2011, ApJL, 740, L16

Lorimer, D. R., \& Kramer, M. 2012, Handbook of Pulsar Astronomy (Cambridge: Cambridge Univ. Press)

Lyubarsky, Y., Eichler, D., \& Thompson, C. 2002, ApJL, 580, L69

Marsden, D., \& White, N. E. 2001, ApJL, 551, L155

Mereghetti, S. 2008, A\&ARv, 15, 225

Mereghetti, S., Pons, J. A., \& Melatos, A. 2015, SSRv, 191, 315

Ng, C.-Y., Kaspi, V. M., Dib, R., et al. 2011, ApJ, 729, 131

Olausen, S. A., \& Kaspi, V. M. 2014, ApJS, 212, 6

Pons, J. A., \& Rea, N. 2012, ApJL, 750, L6

Ransom, S. M. 2001, PhD thesis, Harvard Univ.

Ransom, S. M., Cordes, J. M., \& Eikenberry, S. S. 2003, ApJ, 589, 911

Ransom, S. M., Eikenberry, S. S., \& Middleditch, J. 2002, AJ, 124, 1788

Rea, N., Borghese, A., Esposito, P., et al. 2016, ApJL, 828, L13

Rea, N., \& Esposito, P. 2011, ASSP, 21, 247

Rea, N., Israel, G. L., Pons, J. A., et al. 2013, ApJ, 770, 65

Rea, N., Pons, J. A., Torres, D. F., \& Turolla, R. 2012, ApJL, 748, L12

Scholz, P., Camilo, F., Sarkissian, J., et al. 2017, ApJ, 841, 126

Scholz, P., Kaspi, V. M., \& Cumming, A. 2014, ApJ, 786, 62

Scholz, P., Ng, C.-Y., Livingstone, M. A., et al. 2012, ApJ, 761, 66

Stamatikos, M., Malesani, D., Page, K. L., \& Sakamoto, T. 2014, GCN, 16520, 1

Story, S. A., \& Baring, M. G. 2014, ApJ, 790, 61

Strüder, L., Briel, U., Dennerl, K., et al. 2001, A\&A, 365, L18

Surnis, M. P., Joshi, B. C., Maan, Y., et al. 2016, ApJ, 826, 184

Szary, A., Melikidze, G. I., \& Gil, J. 2015, ApJ, 800, 76

Tendulkar, S. P., Hascöet, R., Yang, C., et al. 2015, ApJ, 808, 32

Thompson, C., \& Duncan, R. C. 1996, ApJ, 473, 322

Thompson, C., Lyutikov, M., \& Kulkarni, S. R. 2002, ApJ, 574, 332

Torres, D. F. 2017, ApJ, 835, 54

Turolla, R., Zane, S., \& Watts, A. L. 2015, RPPh, 78, 116901

van der Horst, A. J., Kouveliotou, C., Gorgone, N. M., et al. 2012, ApJ, 749,122

Verner, D. A., Ferland, G. J., Korista, K. T., \& Yakovlev, D. G. 1996, ApJ, 465,487

Vogel, J. K., Hascoët, R., Kaspi, V. M., et al. 2014, ApJ, 789, 75 
Wadiasingh, Z., Baring, M. G., Gonthier, P. L., \& Harding, A. K. 2017, ApJ, submitted

Weltevrede, P., Johnston, S., \& Espinoza, C. M. 2011, MNRAS, 411, 1917

Wilms, J., Allen, A., \& McCray, R. 2000, ApJ, 542, 914

Woods, P. M., Kaspi, V. M., Thompson, C., et al. 2004, ApJ, 605, 378

Woods, P. M., \& Thompson, C. 2006, in Compact Stellar X-ray Sources, ed. W. Lewin \& M. van der Klis (Cambridge: Cambridge Univ. Press), 547
Yang, C., Archibald, R. F., Vogel, J. K., et al. 2016, ApJ, 831, 80 Younes, G., Gogus, E., Kouveliotou, C., \& van der Hors, A. J. 2015a, ATel, 7213,1

Younes, G., Kouveliotou, C., Kargaltsev, O., et al. 2012, ApJ, 757, 39

Younes, G., Kouveliotou, C., Kargaltsev, O., et al. 2016, ApJ, 824, 138

Younes, G., Kouveliotou, C., \& Kaspi, V. M. 2015b, ApJ, 809, 165

Zhou, P., Chen, Y., Li, X.-D., et al. 2014, ApJL, 781, L16 